Hydroécol. Appl. (2001) Tome 13 Vol. 2, pp. 43-83

\title{
Transfert jusqu'a l'estuaire des radionucléides rejetés par les CNPE du bassin de la Loire
}

\section{Transfer in the Loire river basin down to the estuary of nuclear power plants radioactive discharges}

\section{Françoise Siclet}

EDF R\&D, Département Laboratoire National d'Hydraulique et Environnement, 6 quai Watier, 78401 Chatou Cedex, France

e-mail : francoise.siclet@edf.fr

Résumé. - Les radionucléides d'origine anthropique présents dans le bassin fluvial de la Loire se composent des émissions de centrales électriques d'origine nucléaire, des retombées d'anciens essais d'armes nucléaires et de l'accident de Tchernobyl.

Depuis 1963, le nombre de ces centrales nucléaires a régulièrement augmenté, atteignant en l'an 2000, 14 unités implantées sur 5 sites différents, dont 4 sur la Loire ellemême et le dernier sur son affluent la Vienne. Chacune de ces centrales procède périodiquement à des rejets radioactifs résiduels de faible niveau dans la Loire, à l'origine d'une exposition dosimétrique très faible à proximité des sites, ainsi que le montrent les calculs d'évaluation de l'impact, confirmés par les surveillances environnementales effectuées autour de tous les sites en fonctionnement.

II était toutefois nécessaire d'évaluer dans quelle mesure la succession de plusieurs centrales nucléaires sur le même réseau fluvial était susceptible d'augmenter les concentrations de radionucléides dans l'environnement aval, et d'affecter éventuellement la dose individuelle reçue par la population.

Pour répondre à cette question des effets cumulés à long terme, EDF a lancé en 1998 le "Programme radioécologie Loire », qui a associé trois approches complémentaires comprenant des études sur le terrain, des expériences en laboratoire et des modélisations numériques, en prêtant une attention particulière aux éventuelles accumulations de radionucléides dans les sédiments du fleuve et de son estuaire.

En raison des différences marquées qui distinguent l'environnement d'eau douce du milieu estuarien, chacun de ces deux écosystèmes a fait l'objet d'une étude spécifique.

En ce qui concerne la partie fluviale, les concentrations en radionucléides des phases dissoutes, particulaires et sédimentaires ont été évaluées à l'aide du modèle CRESCENDO, dont le développement et la validation ont nécessité plusieurs étapes en vue de représenter d'abord les phénomènes hydrauliques et de transport par l'élaboration d'un modèle 1D de 350 km de long, associé, lors d'une deuxième étape, aux éléments suivants :

- un modèle de dynamique des sédiments, étalonné par une étude des zones de décantation des particules fines que sont par exemple les barrages et les rivages, 
- un modèle d'échange de contamination entre l'eau et les particules en suspension, provenant d'expériences en laboratoire réalisées pour les différentes catégories de masses d'eau.

Après chaque étape, les valeurs calculées ont été comparées à des résultats de mesures in situ comme les concentrations quotidiennes de tritium relevées à Angers, limite aval du fleuve, afin de confirmer que le modèle représentait fidèlement les processus impliqués.

Lors de la dernière étape, on a calculé avec le modèle CALVADOS les doses individuelles reçues par la population à différents endroits du cours de la Loire. Les radionucléides pris en compte en raison de leur importance relative dans les rejets liquides des centrales ont été les suivants : tritium, ${ }^{14} \mathrm{C},{ }^{58} \mathrm{Co},{ }^{60} \mathrm{Co},{ }^{110 \mathrm{~m}} \mathrm{Ag},{ }^{134} \mathrm{Cs},{ }^{137} \mathrm{Cs},{ }^{54} \mathrm{Mn},{ }^{124} \mathrm{Sb}$ et ${ }^{131} \mathrm{I}$.

Dans la zone estuarienne, 8 campagnes de prélèvement d'eau, de M.E.S et de sédiments ont été réalisées sur une période de 15 mois. L'analyse radioactive couvrait le ${ }^{14} \mathrm{C}$, le ${ }^{90} \mathrm{Sr}$ et le ${ }^{3} \mathrm{H}$, ainsi que les émetteurs gamma, d'origine naturelle (chaînes de désintégration de l'uranium et du thorium, ${ }^{7} \mathrm{Be}$ et ${ }^{40} \mathrm{~K}$ ) ou artificielle (principalement les isotopes du cobalt et du césium).

En se basant, d'une part, sur les résultats des mesures et, d'autre part, sur les résultats de la modélisation 2D sur 4 marées des échanges et du transport des radionucléides, un modèle simplifié de l'estuaire, MOBHYDYS, a été développé pour décrire le devenir des radionucléides sur de plus longues périodes. II calcule avec un pas de temps journalier le bilan entrée-sortie de l'estuaire et les concentrations moyennes dans l'estuaire. Ce modèle a été appliqué au tritium, au cobalt 60 et au césium 137 sur la période 1994 à 1999. Il a permis d'évaluer, en complément aux 8 campagnes de mesures, les stocks de radionucléides présents dans l'estuaire, les flux expulsés vers l'océan, et la part représentée par les rejets des centrales nucléaires, dans cet inventaire.

Mots clés. - Radionucléide, rivière, estuaire, sédiment, eau, dose reçue par la population.

Abstract. - Anthropogenic radionuclide inputs in the Loire river basin mainly consist of radioactive releases from nuclear reactors as well as fallouts from past nuclear weapon tests and from the Chernobyl accident.

Since 1963, the number of power plants has been steadily increasing to reach, in the year 2000, 14 units operating on 5 different sites, with 4 of them located on the Loire river and the last one on the Vienne river, a tributary. Each of these units carry out low level periodic radioactive liquid discharges in the Loire river, inducing very weak doses for each site, as calculated for impact assessment process, and confirmed afterwards by environmental surveillance during power plant operation.

Nevertheless, it was needed to evaluate to what extent the succession of several nuclear power plants on the same river system could increase the concentrations in radionuclides in the downstream environment, and thus affect the dose to the public. To address this long term and cumulative issue, EDF initiated in 1998 the « Loire river radioecology program ", through three complementary approaches with field surveys, laboratory experiments and numerical modelling. Special attention was given to possible accumulation of radionuclide in river and estuary sediments.

Due to the two very different parts of Loire river basin, two specific studies were performed: one on the freshwater environment, the other on the estuarine environment. As concerns the river part, radionuclides concentrations in the dissolved, particulate and sedimentary forms were evaluated using the CRESCENDO model. The development and validation of this model required a multi-step process to represent, firstly, hydraulics 
\& transport through the design of a $350 \mathrm{~km}$ long 1D basic model, associated, in a second step, with :

- a sediment dynamics model, calibrated through monitoring of settling areas for fine particles such as dams and river shores,

- and contamination exchange model in the different aquatic bodies, representing radionuclide exchange between water and particles derived from laboratory experiments.

After each step, the computed values were compared to measured data-sets, such as the daily tritium concentrations measured in Angers, at the downstream limit of the river, to ensure the model adequately described the processes involved.

In the final step, the CALVADOS model was used to calculate doses to the public at different locations along the Loire river. The following radionuclides were considered because of their relative importance in power plant liquid discharges : tritium, ${ }^{14} \mathrm{C},{ }^{58} \mathrm{Co}$, ${ }^{60} \mathrm{Co},{ }^{110 \mathrm{~m}} \mathrm{Ag},{ }^{134} \mathrm{Cs},{ }^{137} \mathrm{Cs},{ }^{54} \mathrm{Mn},{ }^{124} \mathrm{Sb}$ and ${ }^{131} \mathrm{I}$.

In the estuary zone, 8 sampling campaigns for water, suspended matter and sediments were performed over a 15 months period. Radioactive analysis covered ${ }^{14} \mathrm{C},{ }^{90} \mathrm{Sr}$, ${ }^{3} \mathrm{H}$, as well as the naturally occurring gamma-emitters (uranium and thorium decay chains, ${ }^{7} \mathrm{Be}$ and ${ }^{40} \mathrm{~K}$ ), and the artificial gamma emitters (mainly cobalt and cesium isotopes).

From the results of measurement and 2D modelling over 4 tides of radionuclides exchanges and transport, a simplified model, MOBHYDYS, was developped for describing the evolution of radionuclides over longer periods. The calculation, of the estuary input/output balance and mean concentrations was performed with a daily step.

This model, applied to tritium, cobalt 60 and cesium 137 over the 1994-1999 period, permitted to evaluate, in addition to the 8 measurements campaigns, the stocks of radionuclides present in the estuary, the fluxes expulsed towards the ocean, and the part represented by the releases of nuclear power stations in this inventory.

Keywords. - Radionuclide, river, estuary, sediment, water, individual dose to the public.

\section{INTRODUCTION}

Le bassin fluvial de la Loire, le plus grand de France, constitue un système hydrographique d'environ $120000 \mathrm{~km}^{2}$, soit un cinquième de la superficie du pays. Son débit annuel moyen de $850 \mathrm{~m}^{3} / \mathrm{s}$ en fait l'un des principaux apports européens d'eaux fluviales à l'Océan Atlantique. A son embouchure, la Loire traverse un estuaire macrotidal, caractérisé par la présence d'un bouchon vaseux, qui se forme, à cause des gradients de salinité et de densité, dans la zone de mélange entre l'eau douce et l'eau salée. Pouvant retenir jusqu'à 1 million de tonnes de sédiments en suspension, l'estuaire de la Loire est considéré comme une zone potentielle d'accumulation des polluants.

Le bassin de la Loire, en raison de la ressource en eau de refroidissement qu'il représente et de sa position géographique, est le siège d'un fort développement de l'énergie nucléaire. En effet, depuis 1963, année de la mise en service de la première centrale nucléaire sur la Loire, la capacité des centres de production 
d'électricité (CNPE) a augmenté au fil du temps et de l'eau pour atteindre, en 2000, $14500 \mathrm{MWe}$, répartis sur 4 sites en bord de Loire et un site en bord de Vienne.

Grâce à des traitements importants des effluents et une politique active de réduction des émissions dans l'environnement, chacune de ces installations ne rejette périodiquement, dans la limite de normes contraignantes, qu'une faible quantité d'effluents radioactifs dans les cours d'eau et l'atmosphère. Les calculs de doses et les études de surveillance de l'environnement ont montré que l'impact de ces rejets, à l'échelle d'un site, est très faible. Cependant, la présence de plusieurs installations sur un même bassin nécessitait de vérifier l'effet du cumul de leurs rejets dans l'environnement et les conséquences éventuelles en terme de doses pour le public.

Pour répondre à cette préoccupation, EDF a lancé, en 1998, un important programme de recherche visant à s'assurer que les objectifs réglementaires étaient respectés sur l'ensemble du bassin de la Loire, même pour des situations hydrauliques extrêmes.

Ont été étudiés en particulier les périodes de faibles débits et les phénomènes de dépôts sédimentaires dans les zones à faible vitesse d'écoulement, entraînant une accumulation de radionucléides sur de longues périodes en un point donné, avant d'être remis en suspension lors des crues; de même, l'étude a porté sur le bouchon vaseux de l'estuaire, constituant un lieu de piégeage et d'accumulation des radionucléides sur des durées d'une à plusieurs années.

Pour analyser ces questions, il importe de faire la distinction entre les formes dissoutes et particulaires, dans la mesure où les premières sont transportées plus rapidement vers l'aval et l'océan que les formes particulaires, soumises à de nombreux épisodes de dépôt et de remise en suspension. Compte tenu de la difficulté à quantifier la spéciation et le transport, en raison de la séquence complexe d'interactions physiques et biogéochimiques qui se produit le long du cours du fleuve et dans l'estuaire, l'approche adoptée a été celle de la simulation numérique, combinée à des expériences en laboratoire et à des études de terrain destinées à étalonner et à valider les modèles.

La partie expérimentale a permis de connaître la nature et les cinétiques des réactions auxquelles participent les radionucléides; elle a été menée en collaboration avec le CEREGE (Centre Européen de Recherche et volet d'Enseignement en Géoscience de l'Environnement). Les mesures in situ, conduites avec le CNRS et le CEA, avaient pour objectifs d'identifier les phénomènes déterminants dans le transport des sédiments et des radionucléides associés et de constituer une base de données permettant la validation de l'outil de simulation.

La simulation a porté sur une période de six ans de 1994 à 1999 pour 


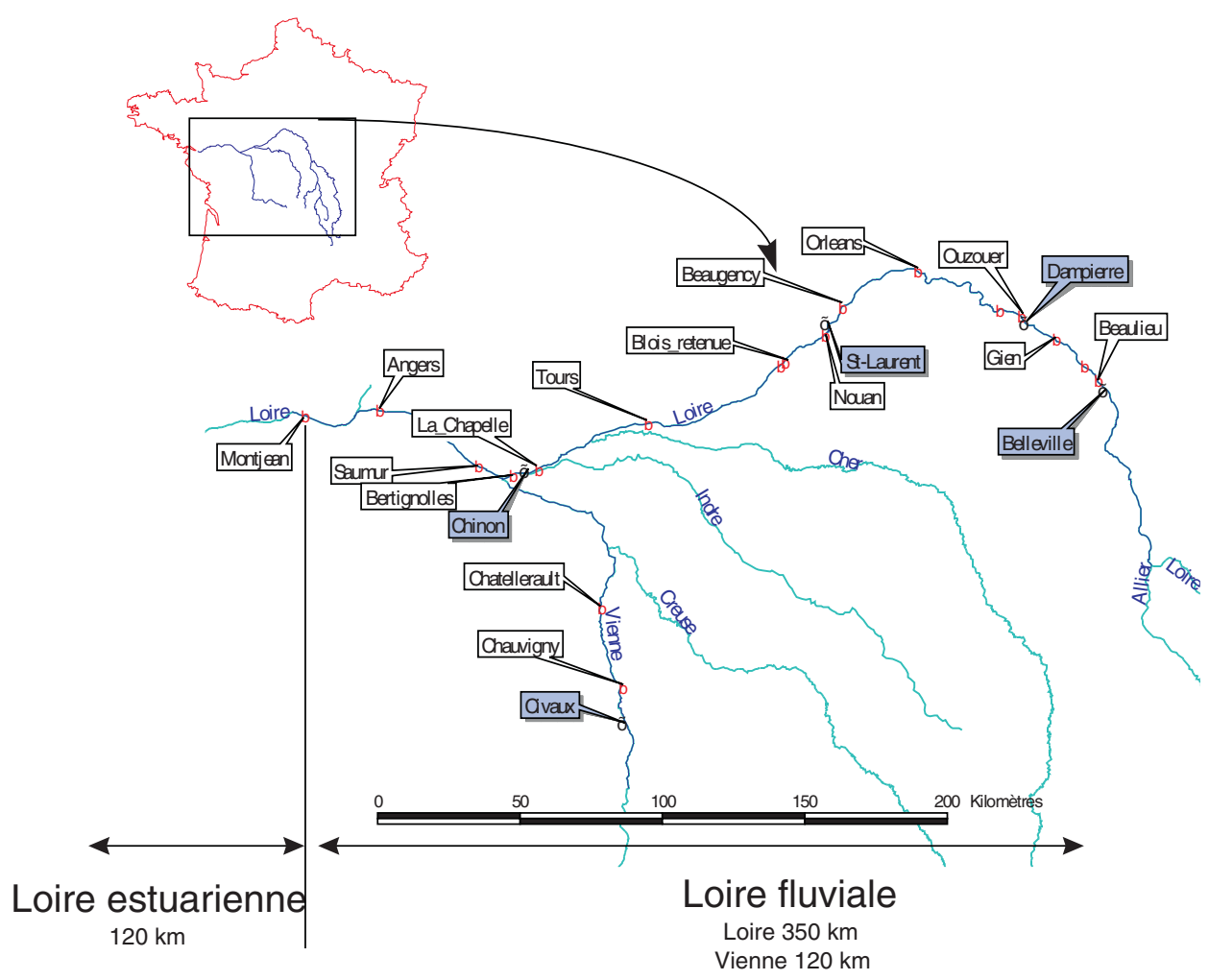

Fig. 1. - Le réseau hydrographique de la Loire : localisation de la zone d'étude.

Les biefs modélisés sont représentés par un trait gras, les centrales sont indiquées sur fond gris et les agglomérations où les doses à l'homme ont été calculées sont indiquées sur fond blanc.

Fig. 1. - The Loire river system : location of study area.

Reaches included in this study are represented by bold lines, power plants have gray labels and locations where dose to the public are calculated have white labels.

couvrir une gamme étendue de situations hydrologiques. Elle comporte trois modules reflétant les disciplines concernées : la modélisation hydraulique, la modélisation du transport sédimentaire et la modélisation radioécologique. A chaque étape du développement de l'outil de simulation, les résultats de calcul ont été comparés aux mesures dans l'environnement pour s'assurer que le mo- dèle décrivait correctement les processus concernés.

Les calculs ont porté sur les principaux radionucléides présents dans les effluents radioactifs liquides, à savoir H-3, C-14, Cs-137, Cs-134, Co58, Co-60, Mn-54, Sb-124, I-131, Ag$110 \mathrm{~m}$. Le Sr-90, bien que rarement détecté dans les rejets des centrales à eau pressurisée, a été ajouté à cette liste car il fait partie des radionu- 
cléides présents dans l'environnement suite aux retombées des anciens tirs nucléaires atmosphériques.

Les variables calculées sont :

- les concentrations en radionucléides dans l'eau et les sédiments de la Loire et de l'estuaire résultant des rejets radioactifs réels des CNPE de Belleville, Dampierre, Saint-Laurent, Chinon et Civaux ;

- les concentrations en radionucléides dans les compartiments de l'écosystème aquatique et terrestre, en amont et en aval de chaque centrale et au niveau de chaque grande agglomération, le long de la Loire et de la Vienne ;

- les doses annuelles à l'homme, aux mêmes stations que précédemment, pour chacune des années.

Compte tenu de la spécificité des deux entités hydrographiques concernées (cours d'eau et estuaire), des démarches différentes de modélisation ont été adoptées dans chacun de ces cas.

\section{TRANSFERT DES RADIONUCLÉIDES EN LOIRE FLUVIALE}

La Figure 1 représente le bassin fluvial de la Loire, avec ses affluents et les cinq sites de centrales nucléaires d'EDF.

Pour pouvoir couvrir de longues durées, différents régimes de débit et tous les lieux intéressants le long du bassin, deux modèles numériques ont été utilisés :

- le modèle CRESCENDO élaboré pour évaluer les concentrations de radionucléides dans les phases dissoute, particulaire et sédimentaire,

- et le modèle CALVADOS pour calculer les concentrations le long de la chaîne alimentaire et la dose individuelle reçue par le public.

Le développement et la mise en œuvre de ces modèles a nécessité non seulement d'assembler des bases de données sur les débits, les charges en matières en suspension, les rejets radioactifs et de réaliser des expériences radioécologiques spécifiques au fleuve (pour déterminer, par exemple, le $\mathrm{Kd}$ et les paramètres cinétiques des échanges de radionucléides entre l'eau et les matières en suspension), mais également de mener un travail complémentaire de collecte de données de terrain, centré sur les domaines suivants :

- mesures détaillées dans le temps des matières en suspension, avec des prélèvements d'eau toutes les 8 heures, pendant plusieurs mois en 1998-1999, dans la Loire, à hauteur de Belleville (limite amont de la zone d'étude), et également à hauteur d'Angers (limite aval), avec des mesures de concentration en MES (matières en suspension) sur les composites journaliers quand les débits sont stables et sur chaque échantillon quand les débits augmentent ; 
- étude des dépôts et de l'érosion à des emplacements sélectionnés, en particulier au niveau de la retenue de Blois ;

- étude des concentrations de radionucléides dans l'eau à Angers et dans les sédiments, dans les zones de dépôt fin le long de la Loire ;

- étude des pratiques agricoles dans chaque département traversé.

La présentation qui suit expose les caractéristiques des deux modèles employés pour la partie fluviale et les résultats tirés d'une simulation sur six ans.

\subsection{Le modèle CRESCENDO}

Un ensemble de modules ont été assemblés dans le système baptisé CRESCENDO (Concentrations en Radionucléides dans l'Eau et les Sédiments Cumul sur l'ENsemble du DOmaine) que l'on peut diviser en deux catégories: les sous-modèles couplés et les sous-modèles découplés (Fig. 2). Les sous-modèles couplés utilisés sont les suivants :

- le code hydrodynamique LIDO, code 1D non permanent, qui résout les équations d'écoulement à

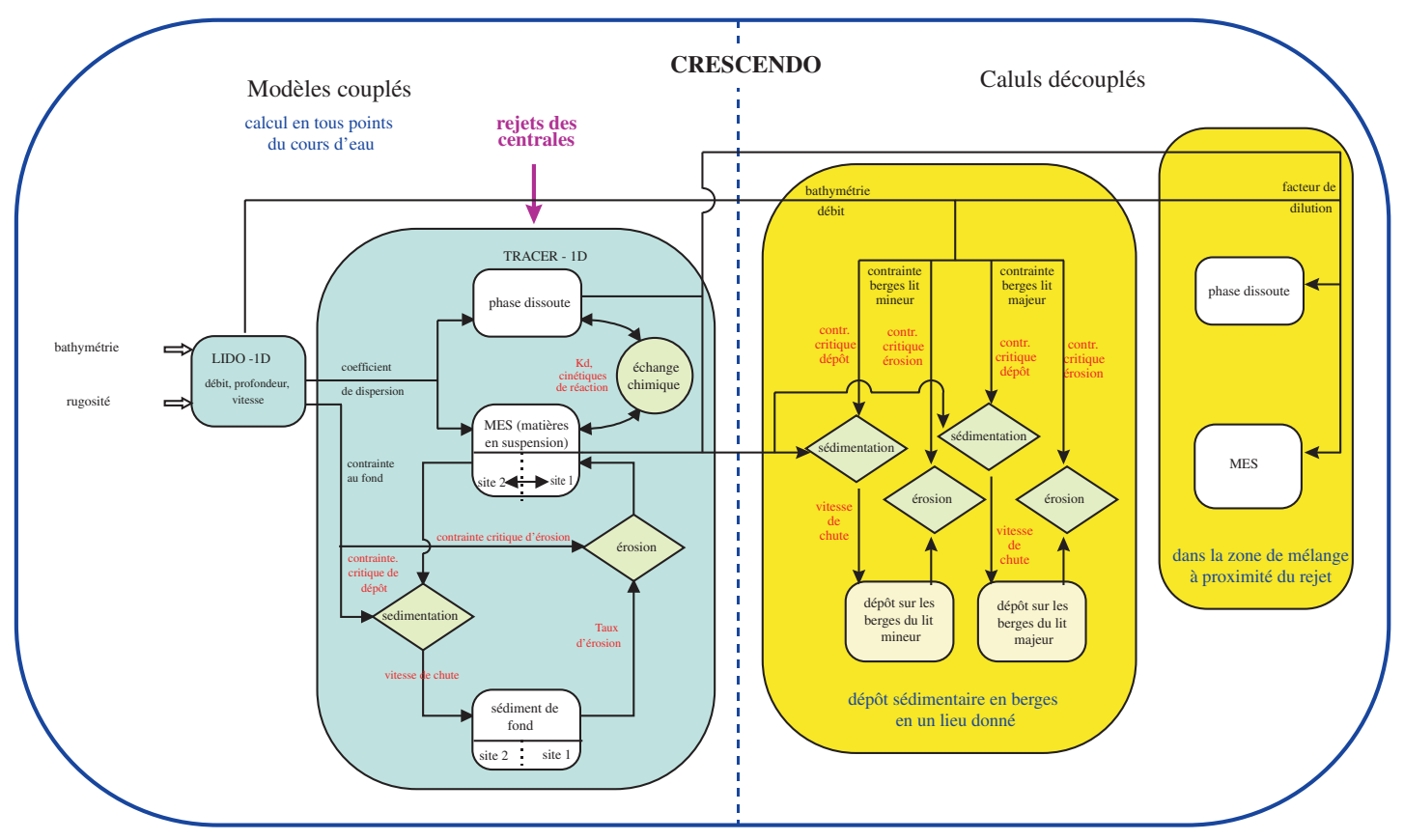

Fig. 2. - Structure du modèle CRESCENDO.

Fig. 2. - Structure of the CRESCENDO model. 
surface libre. La topographie du fleuve a été prise en compte par LIDO, grâce à des données bathymétriques très précises sous forme de profils en travers espacés d'environ $1 \mathrm{~km}$;

- le module de transport TRACER qui utilise les résultats du modèle hydraulique pour simuler le transport 1D et la dispersion des substances dissoutes ou des solides en suspension. Les phénomènes de sédimentation et d'érosion sont décrits par les lois de Krone et Partheniades, gouvernées par deux paramètres: la vitesse de chute des particules et le taux d'érosion. Les contraintes critiques de dépôt et d'érosion ont été calées à partir des mesures effectuées sur la retenue de Blois ;

- le module d'échanges de radionucléides qui décrit les échanges entre la phase dissoute et la phase particulaire. Ces échanges sont modélisés par des cinétiques correspondant à deux réactions réversibles successives qui tendent vers l'équilibre, caractérisé par le $\mathrm{Kd}$, coefficient de distribution. Des expériences ont été conduites sur site et en laboratoire afin de déterminer ces paramètres pour la Vienne et la Loire, en conditions estivales et hivernales.

Ces codes sont intégrés dans la structure informatique PLATINEA développée par EDF R\&D.

Le dépôt de sédiments fins sur les berges n'est pas inclus dans le modèle de transport couplé car ce type de dépôt, très localisé sur les rives de la Loire, n'a pas d'influence sur le bilan de masse en radionucléides dans la rivière. L'approche retenue, pour pouvoir néanmoins prendre en compte ce processus dans les voies d'exposition de l'homme par irradiation externe, a été de développer des méthodes de calcul découplées qui utilisent les résultats des modèles couplés pour estimer les dépôts sédimentaires en berge. La même démarche a été adoptée pour calculer les concentrations en radionucléides dans la zone de mélange à proximité des points de rejet des effluents radioactifs.

\subsection{La modélisation hydraulique et le transport des substances dissoutes}

\subsubsection{Etalonnage et validation des modèles hydraulique et de transport}

Le calage des coefficients de Strickler a été effectué à partir d'une base de données très détaillée des lignes d'eau dans des conditions d'étiage, de débits moyens et forts, compilée par la DIREN Centre. La comparaison des vitesses calculées par le modèle aux vitesses estimées à partir de plusieurs campagnes de traçage a permis de valider la modélisation numérique.

Pour calibrer le coefficient de dispersion et valider le modèle de transport des substances dissoutes, des échantillons d'eau ont été prélevés toutes les 8 heures dans la partie aval du bassin fluvial (dans la ville d'Angers), et leur concentration en tri- 


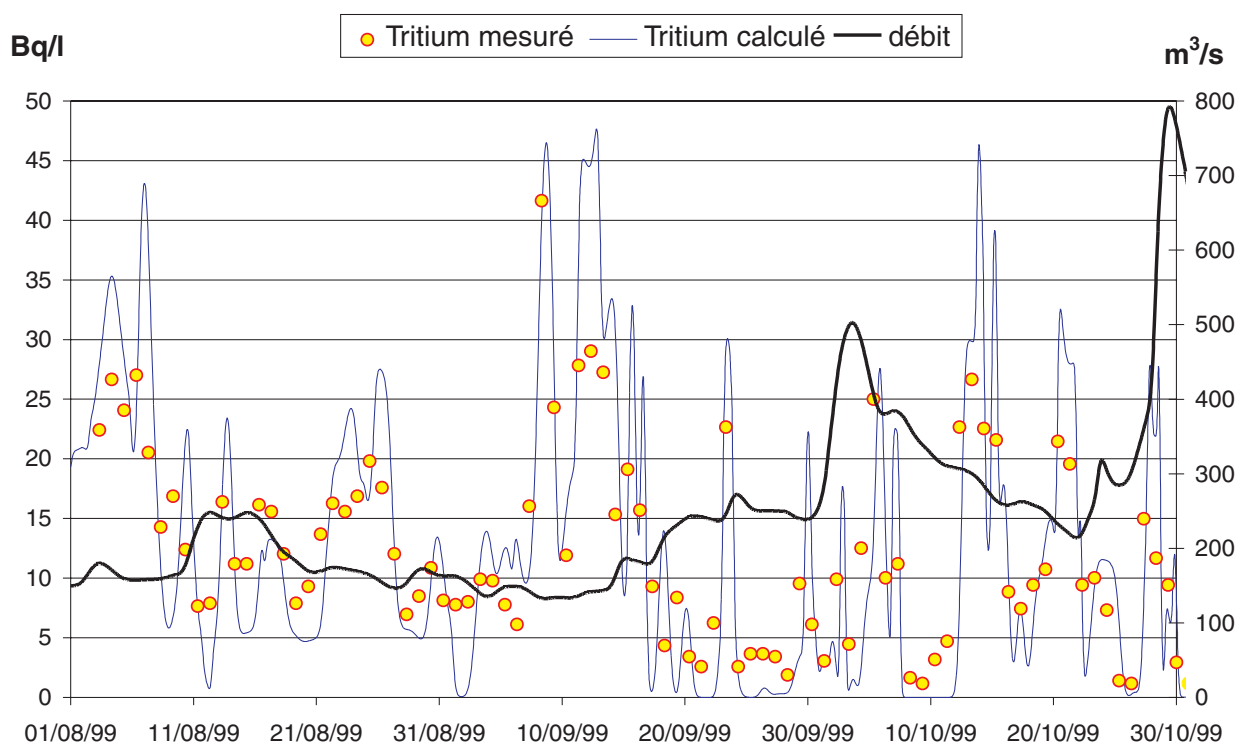

Fig. 3. - Concentrations en tritium dans la Loire à Angers. Comparaison calculs-mesures.

Fig. 3. - Observed and calculated values of tritium concentrations in the Loire river, in Angers.

tium a été analysée. La Figure 3 donne un exemple de validation dans lequel les calculs sont comparés aux valeurs observées.

\subsubsection{Simulation des concentrations} en tritium le long de la Loire et de la Vienne

A partir des rejets réels de chaque centrale sur le bassin de la Loire, les concentrations horaires en chaque point de la Loire et de la Vienne ont été calculées de 1994 à 1999. Les concentrations moyennes en tritium augmentent le long du cours de la Loire depuis le CNPE de Belleville jusqu'à celui de Saint-Laurent : cette partie du fleuve reçoit les effluents de trois centrales électriques sans apport d'eau significatif de la part des affluents. Les concentrations les plus élevées sont observées dans le tronçon de $100 \mathrm{~km}$ qui s'étend entre le CNPE de Saint-Laurent et le confluent avec le Cher, à Tours. Après une forte diminution en aval de ce confluent, les concentrations en tritium atteignent un pic dans le tronçon de $7 \mathrm{~km}$ qui sépare le CNPE de Chinon et le confluent avec la Vienne, pour diminuer ensuite jusqu'à l'estuaire (Fig. 4). Ces tendances varient peu d'un mois à l'autre alors qu'on constate en un lieu donné de fortes variations des concentrations d'un mois à l'autre en fonction du débit d'eau et de la gestion des effluents radioactifs. L'exemple de juillet 1997 présenté sur la Figure 4 correspond à une période où, en raison d'un faible débit, les centrales n'ont pas été autorisées à relâcher leurs effluents, ou n'ont pu le faire que très modéré- 


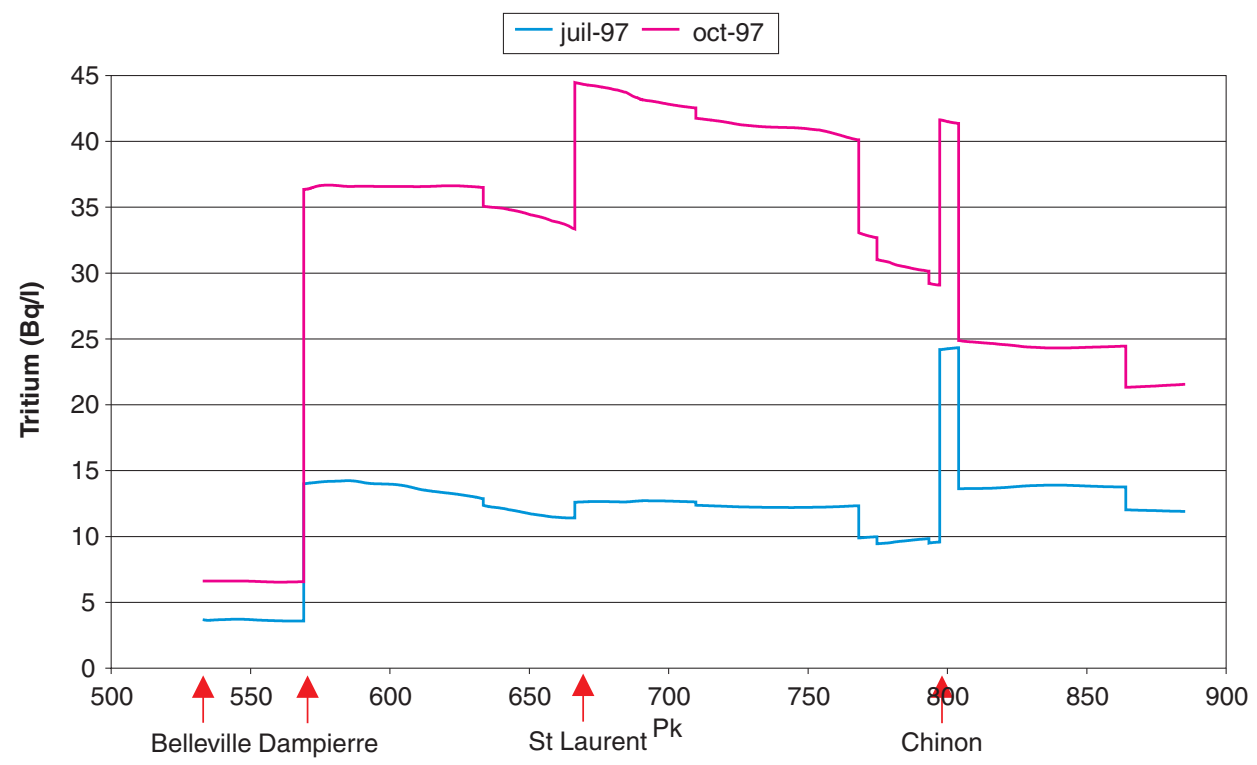

Fig. 4. - Evolution des concentrations en tritium le long de la Loire (moyenne des concentrations horaires en juillet 1997 et octobre 1997).

Fig. 4. - Spatial evolution of tritium concentrations along the Loire river (average of hourly concentrations in July 1997 and October 1997).

ment, ce qui les a conduites à les stocker jusqu'en octobre, en attendant de pouvoir les évacuer dans des débits plus importants. Sur l'ensemble des six années simulées, les concentrations mensuelles moyennes les plus faibles s'observent en janvier et en février. Elles sont alors de quatre à cinq fois inférieures à celles des mois d'août à octobre.

\subsection{La modélisation sédimentaire et le transport des substances associées aux particules}

\subsubsection{Etalonnage des paramètres de sédimentation et d'érosion}

Le barrage mobile de Blois, I'un des rares endroits du cours intermé- diaire de la Loire où se produit une décantation des particules fines, a été retenu comme site d'étude du transport des sédiments. Ce barrage est dressé chaque été afin d'entretenir un niveau d'eau constant propice aux loisirs nautiques (Photo 1).

Compte tenu de l'hétérogénéité transversale et longitudinale du dépôt de sédiments, un modèle numérique bidimensionnel TELEMAC-2D a été employé, couplé avec SUBIEF-2D, pour résoudre les équations d'écoulement en eaux peu profondes, de transport et de dispersion. Cet outil permet également de calculer les flux de sédimentation et d'érosion des matières en suspension.

Le calage du modèle 2D s'est appuyé d'une part, sur les campagnes 


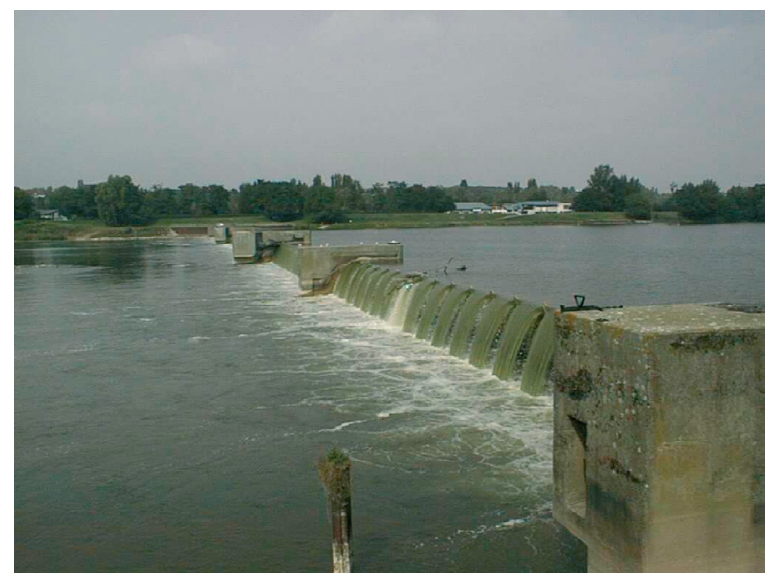

Photo 1. - Barrage de Blois en position haute.

Photo 1. - The Blois dam in high position. de mesures réalisées durant l'été 1999 pour caractériser l'épaisseur des dépôts à partir de carottages, d'autre part sur le suivi des concentrations de MES en amont et en aval de la retenue lors de l'épisode de remise en suspension du 26 octobre 1999, au moment de l'abaissement du barrage. Les résultats fournis par ce modèle (Fig. 5) ont ensuite servi à caler le sous-modèle de sédimentation 1D (Fig. 6) et les calculs de dépôt sur les berges du modèle CRESCENDO, présentés au paragraphe 2.3.3.

\subsubsection{Cinétiques d'adsorption et de désorption des radionucléides}

Les échanges de radionucléides à l'interface solide-liquide sont décrits par un système de deux réactions successives réversibles: une première réaction correspondant à un échange d'ions avec des sites non spécifiques en surface des particules, une deuxième réaction plus lente faisant intervenir des sites spécifiques (fixation à l'intérieur des feuillets d'argiles, oxydation...). Les paramètres descriptifs des cinétiques de chacune des réactions pour chacun des radionucléides ont été déterminés à partir d'expérimentations de marquage, en laboratoire, d'échantillons d'eau et de MES prélevés en Loire et en Vienne. L'influence, sur les échanges, des variations saisonnières de la nature des MES a conduit à appliquer dans le modèle, pour le cobalt, le césium, le manganèse et l'argent, des cinétiques différentes en été (avril à septembre) et en hiver (octobre à mars).

L'iode, l'antimoine et le strontium présentent en revanche des cinétiques d'échange très rapides identiques d'une saison à l'autre. On a donc considéré que le comportement de ces radionucléides ne nécessitait de prendre en compte ni les cinétiques d'échange (détermination d'un coefficient de distribution à l'équilibre) ni les variations saisonnières. 


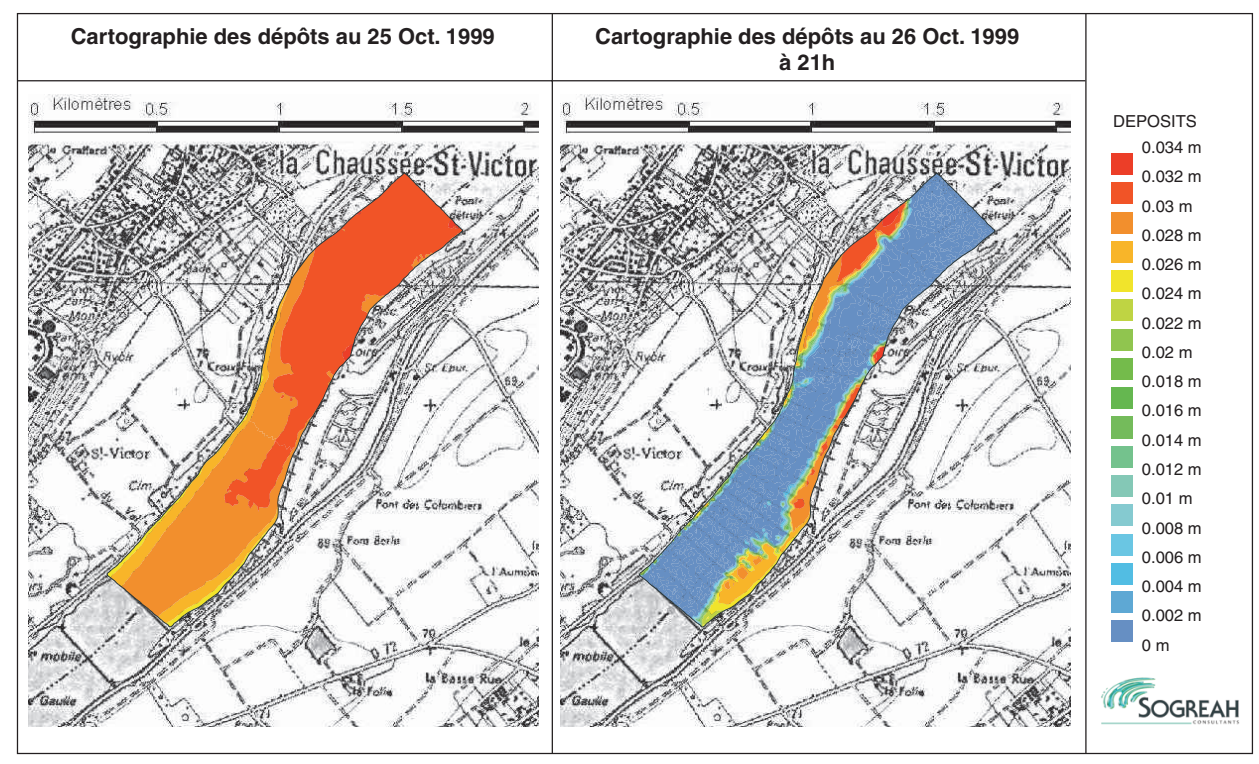

Fig. 5. - Cartographie des dépôts de sédiments dans la retenue de Blois, calculés avec le modèle 2D, le jour précédant l'abaissement du barrage (à gauche), et 6 heures après l'abaissement du barrage (à droite).

Fig. 5. -2D-model calculated sediment deposit in the Blois reservoir, the day before the lowering of the dam (left) and 6 hours after the lowering of the dam (right).

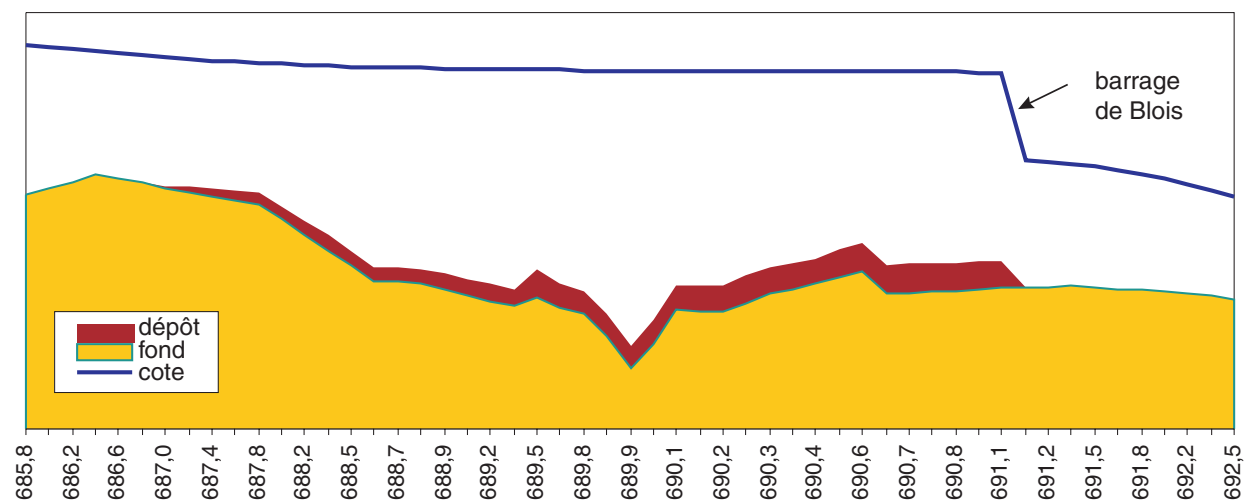

\section{Pk (km)}

Fig. 6. - Cartographie avec le modèle 1D des dépôts dans la retenue de Blois le 26/10/99 à $15 \mathrm{H}$.

Fig. 6. - 1-D model sediment deposit in the Blois reservoir, on Oct 26,1999 at 3 p.m. 
Pour valider les modèles d'échange, les cinétiques issues d'expérimentations en laboratoire ont été comparées aux résultats obtenus en Loire lors de suivis de rejets radioactifs. Trois campagnes engagées sur la Loire, pendant des périodes de rejet radioactif, ont permis d'évaluer la répartition des radionucléides entre la phase dissoute et la phase particulaire. Elles se sont déroulées en aval du CNPE de Dampierre, en Juillet 1993, et Mars 1994, et en aval du CNPE de Chinon, en Juin 1998. La confrontation des résultats obtenus in vitro et in situ (Fig. 7) montrent que les coefficients de distribution obtenus en Loire pour le cobalt et l'argent ont tendance à dépasser les valeurs en laboratoire alors que l'on constate l'inverse pour le césium. On note cependant que malgré ces variations, les valeurs in situ et in vivo sont du même ordre de grandeur. En revanche, pour le manganèse, la seule mesure in situ ne permet pas de valider les résultats expérimentaux.

\subsubsection{Validation des calculs de dépôt sur les rives}

La méthode mise en œuvre dans CRESCENDO pour l'évaluation des dépôts en berges s'appuie sur la décomposition de la zone des dépôts en :

- une zone de dépôt sur les berges du lit mineur, nettoyée dès que la rivière déborde dans le lit majeur,

- une zone de dépôt sur les berges du lit majeur, existant tant que le débit ne dépasse pas une valeur critique pour laquelle les contraintes sur le fond, même sur les berges, deviennent trop importantes; le dépôt n'est alors plus possible, et l'érosion se met en place.

Le dépôt et l'érosion sont évalués à partir d'un modèle « à seuils » (analogue à la formule de Krone), basé sur une vitesse de chute et une contrainte critique de dépôt, identiques pour toutes les berges de la Loire. Pour chaque station, la contrainte de cisaillement sur le fond est répartie transversalement le long du profil en considérant qu'en chaque point, la contrainte est proportionnelle à la hauteur d'eau. La formule utilisée est la suivante :

$$
\begin{aligned}
\tau_{b}(x, y)=\overline{\tau_{b}} \cdot \frac{\left(\bar{c}-z_{x, y}\right)}{\bar{R}_{h}} & = \\
& \rho \cdot g \frac{\left(\bar{c}-z_{x, y}\right) \cdot \bar{v}^{2}}{\operatorname{str}^{2} \bar{R}_{h}^{4 / 3}}
\end{aligned}
$$

où :

$\tau_{b}(x, y)$ contrainte de cisaillement sur le fond, au point de coordonnées $(x, y)$ de la section considérée

$\overline{\tau_{b}}$ contrainte moyenne de cisaillement sur le fond, pour la section considérée

$\rho$ masse volumique de l'eau

$g$ accélération de la pesanteur

$\bar{c}$ cote de la surface libre, calculée avec LIDO

$z_{x, y}$ altitude $z$ du point $(x, y)$ du profil ; $\left(\bar{c}-z_{x, y}\right)$ correspond alors à la hauteur d'eau au niveau du point $(x, y)$

$\bar{v}$ vitesse moyenne sur la section considérée, calculée avec LIDO 

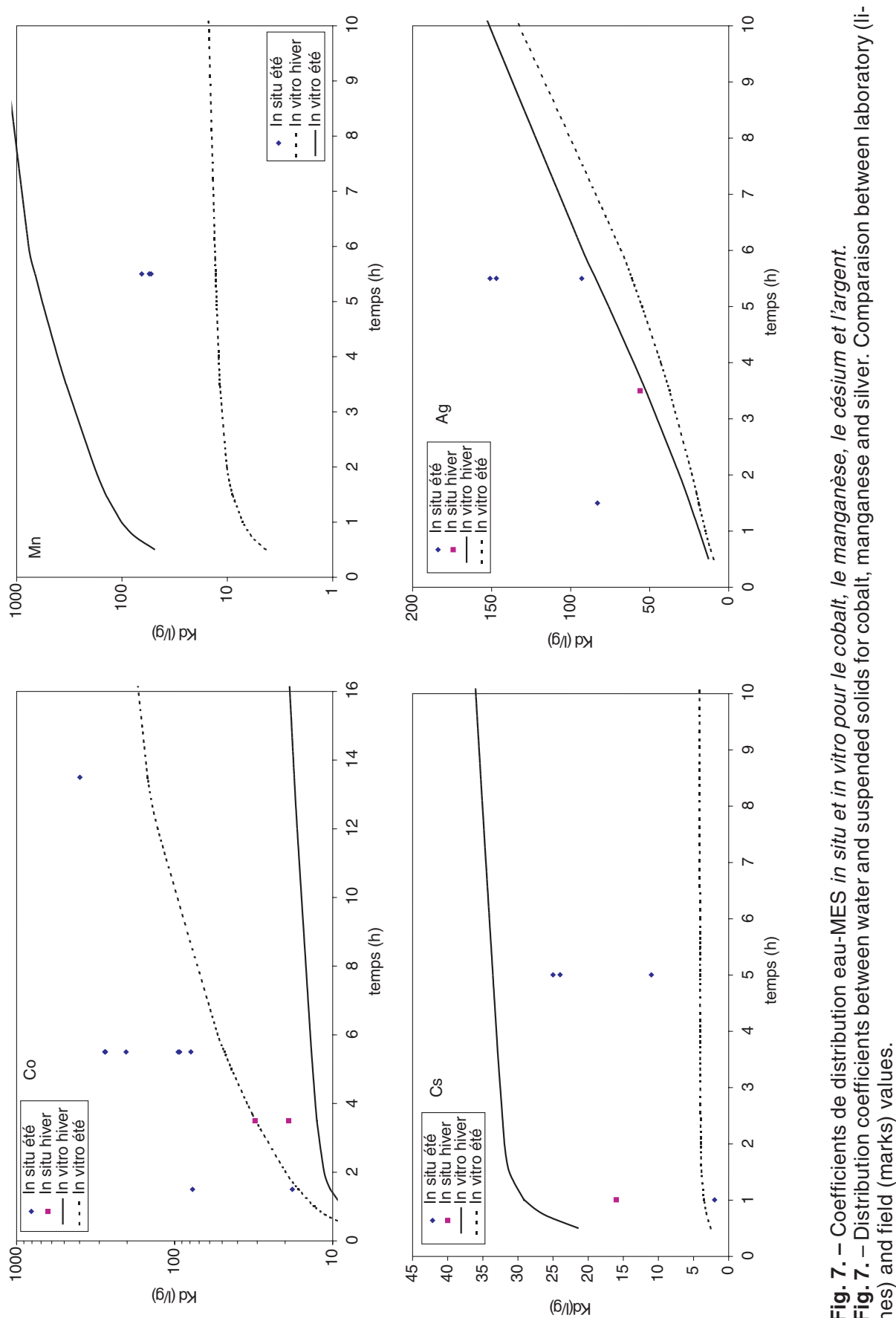

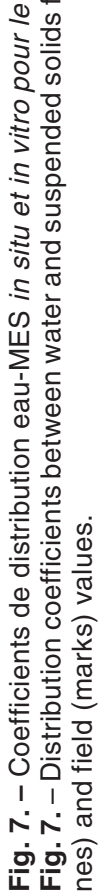



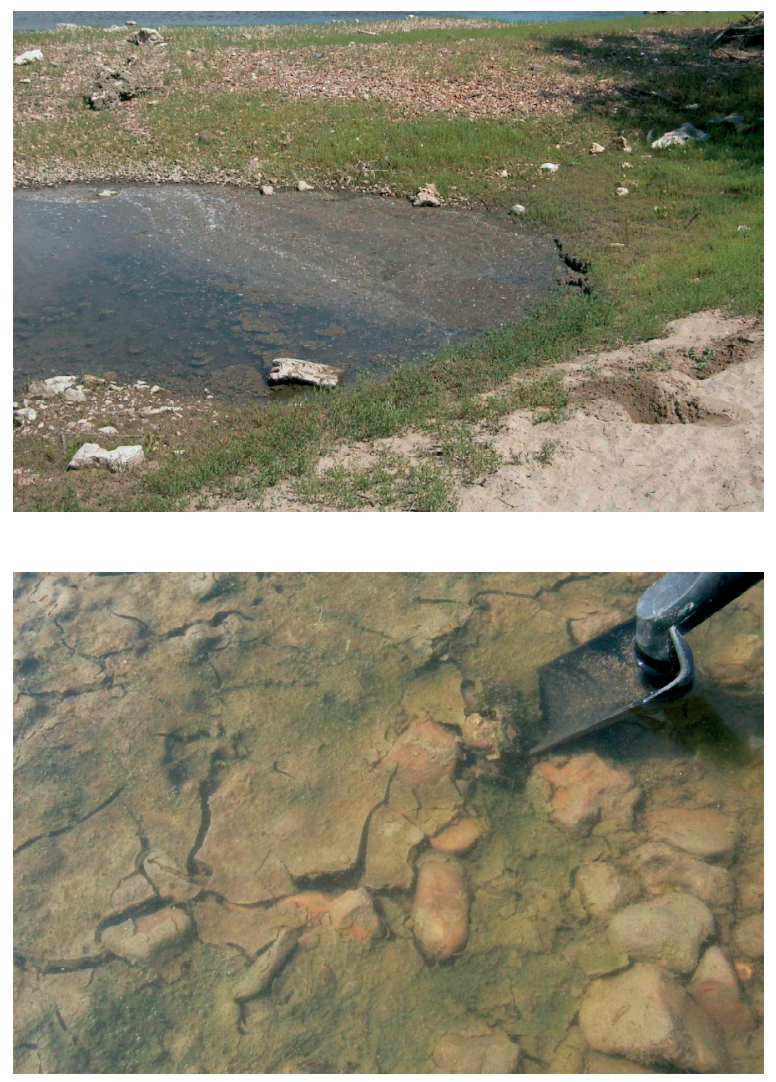

Photos 2 et 3. - Exemple de zone de sédimentation en berges de la retenue de Blois (bras mort). Photo 2 : graviers à l'arrière-plan, sables au premier plan. Photo 3 : zoom sur sédiments fins de la zone immergée.

Photos 2 et 3. - Example of settling area along the Blois reservoir shores (backwaters). Photo 2: gravels in the background, sand in the foreground. Photo 3 : zoom on fine sediment in the immerged zone.

\section{Str coefficient de Strickler}

$\overline{R_{h}}$ rayon hydraulique moyen sur la section considérée, calculé avec LIDO

La contrainte critique de dépôt en berges a été déterminée de telle façon à respecter les observations de terrain relatives à la localisation des zones de sédimentation fine (Photos 2 et 3). La valeur choisie correspond au maximum des valeurs relevées, à savoir la valeur obtenue en berges à hauteur de Bertignolles. II a été supposé, d'autre part, que l'érosion se déclenchait dès que la contrainte sur le fond dépassait la contrainte critique de dépôt.

La vitesse de chute a été calée sur la retenue de Blois, puisque l'on disposait des hauteurs de sédiments sur les berges de la retenue pour chaque mois de l'été 1999, données issues de la modélisation hydro-sédimentaire $2 \mathrm{D}$ décrite au $\S 2.3 .1$.

Les valeurs prédites de dépôt sur les rives ont été comparées avec les concentrations observées dans les sédiments prélevés dans les berges à 

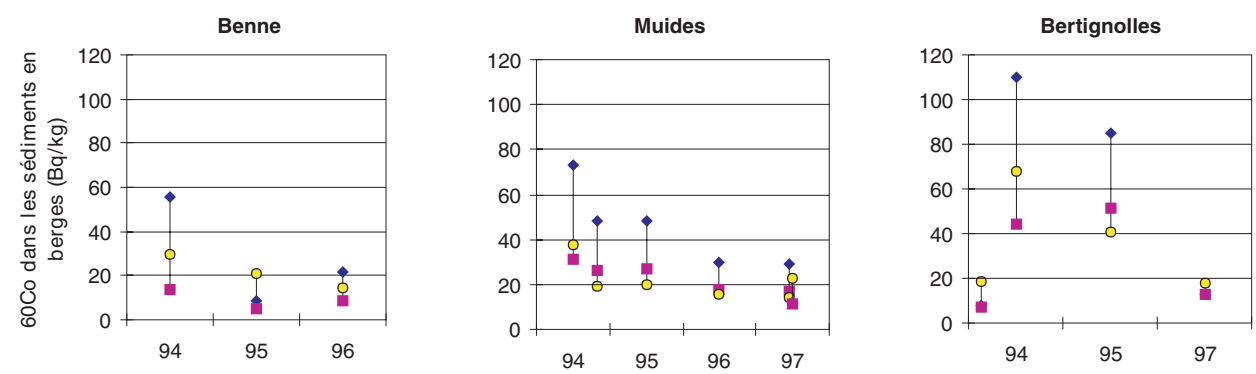

- mesures normalisées à la teneur en argile

- mesures normalisées à la teneur en argiles+limons fins

o résultats des calculs de dépôt en berges

Fig. 8. - Comparaison calculs-mesures pour les dépôts de cobalt 60 dans les sédiments des berges.

Fig. 8. - Comparison of predicted and observed cobalt 60 concentrations in shore sediment.

l'occasion des suivis radioécologiques annuels de chaque site de centrale. Etant donné que le modèle calcule l'activité liée aux particules fines, les valeurs observées ont été corrigées en faisant l'hypothèse que la radioactivité présente dans un échantillon de sédiment n'est associée qu'à la partie constituée d'argile et/ou de vase fine. La Figure 8 présente les concentrations de cobalt 60 dans les sédiments de berge, et montre que les résultats du modèle s'inscrivent dans la plage des concentrations observées.

\subsubsection{Simulation des dépôts de 1994 à 1999}

Les variations saisonnières ont une influence notable sur l'amplitude des dépôts avec un minimum pen- dant les périodes de forts débits de Novembre à Avril et des valeurs augmentant régulièrement de Mai à Septembre (Fig. 9).

Les sédiments de fond sont totalement remis en suspension chaque année. Il en est de même pour les dépôts sur les berges dans la plupart des stations (Fig. 10), sauf à Blois où la remise en suspension est partielle et ne concerne que le lit mineur. II y a donc, au niveau de la retenue de Blois, une accumulation des dépôts sur les berges du lit majeur sur plusieurs années (Fig. 9). Pour les autres stations, le dépôt maximal sur les berges est observé en octobre 96 . Ce pic intervient après un été où les concentrations moyennes dans la rivière ont été les plus élevées des six étés simulés. 


$\rightarrow-\operatorname{Co60}$ SED $(\mathrm{Bq} / \mathrm{m} 2) \rightarrow$ Co60 MES $(\mathrm{Bq} / \mathrm{kg}) \rightarrow$ Dépôt en berge $(\mathrm{Bq} / \mathrm{m} 2)$

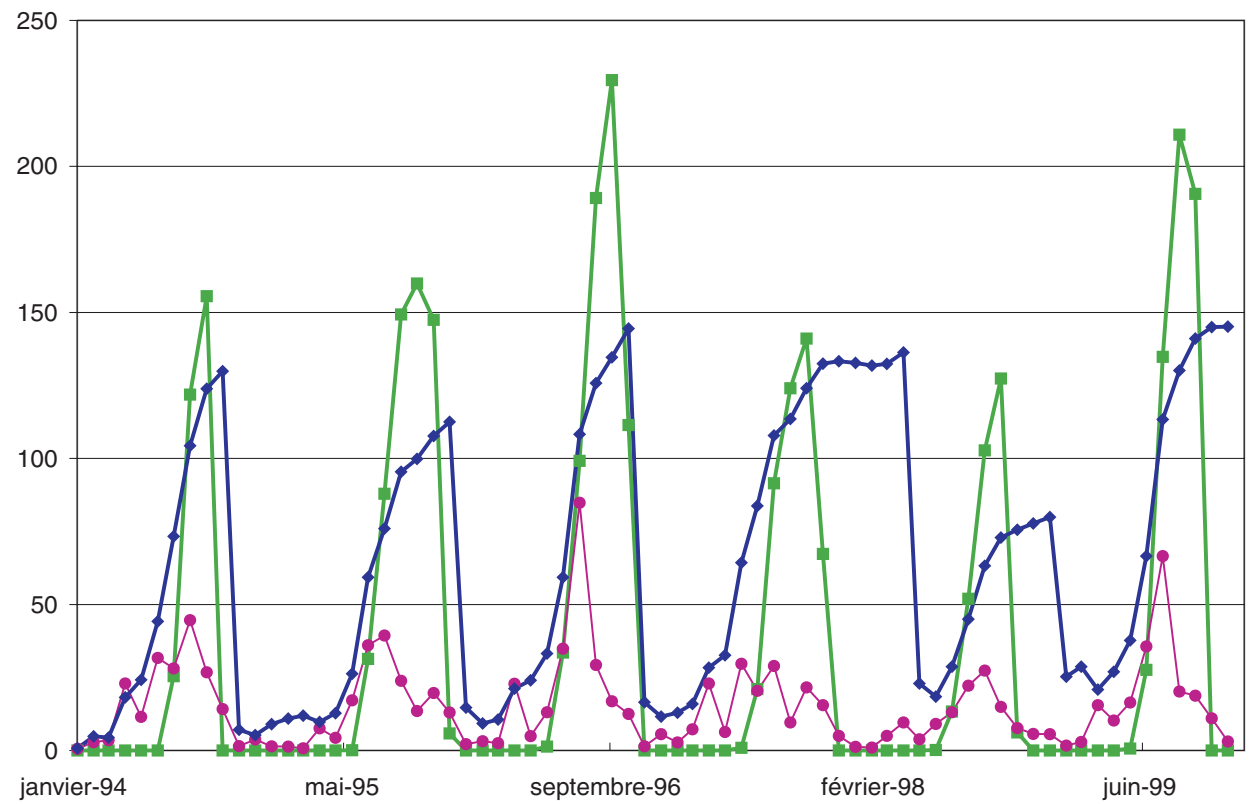

Fig. 9. - Concentrations en Cobalt 60 dans les MES, les sédiments de fond et sur les berges de la retenue de Blois.

Fig. 9. - Cobalt 60 concentrations in Blois reservoir suspended solids, bottom sediment and shore sediment.

On constate une forte variabilité des dépôts sur les berges, d'une station à l'autre, avec des écarts d'un facteur 20 entre les extrêmes (Angers pour le minimum et l'aval immédiat de Saint-Laurent pour le maximum). Les valeurs plus faibles à Bertignolles (aval immédiat du CNPE de Chinon) qu'à Saumur (Fig. 10) illustre le fait que la distribution spatiale des radionucléides dans les dépôts des berges diffère fortement de la distribution dans l'eau (Fig. 11).

Le principal facteur régissant l'activité en radionucléides déposée sur les rives correspond à la topographie locale du lit du fleuve et, dans une moindre mesure, à la concentration des radionucléides dans l'eau.

La simulation des événements de remise en suspension dans la retenue de Blois a permis de tirer les enseignements suivants :

- il ne se produit pas d'augmentation notable de la concentration en radionucléides dissous,

- on constate une augmentation de courte durée de la concentration en radionucléides particulaires (exprimée en $\mathrm{Bq} / \mathrm{m}^{3}$ ) lors de l'érosion 


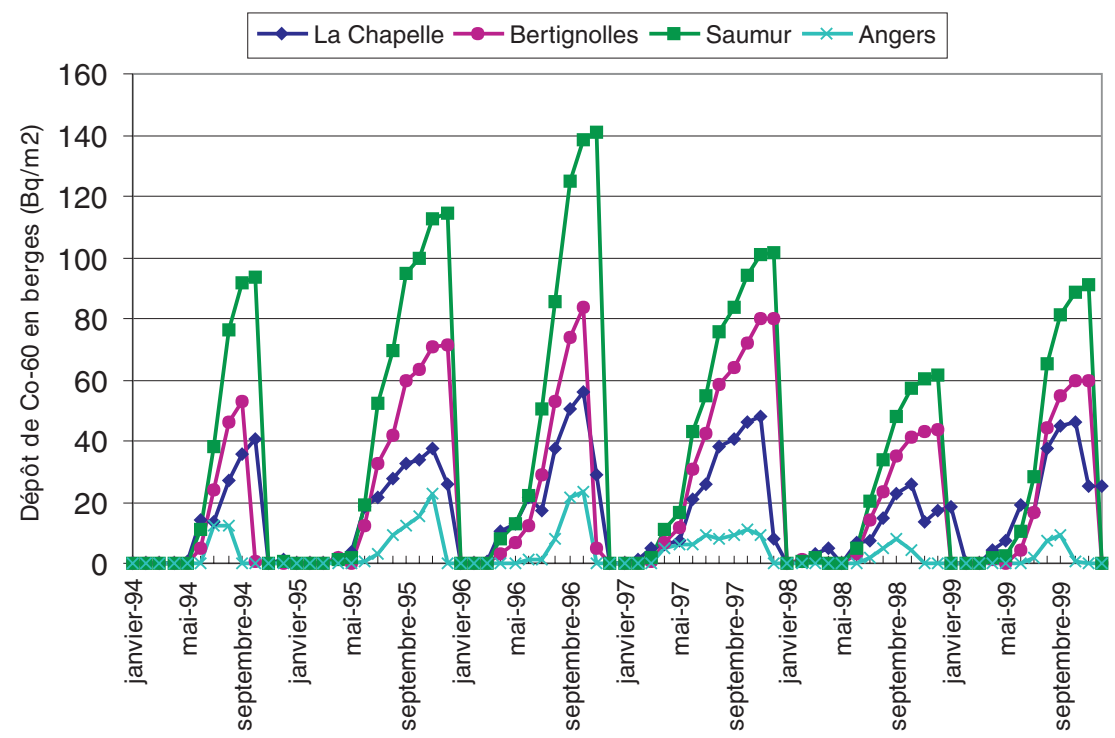

Fig. 10. - Evolution des dépôts de ${ }^{60} \mathrm{Co}$ sur les berges de quatre stations situées entre l'amont de la centrale de Chinon (La Chapelle/Loire) et Angers.

Fig. 10. - Evolution of ${ }^{60} \mathrm{Co}$ shore deposits at 4 stations located between La Chapelle/Loire (upstream of Chinon power plant) and Angers.

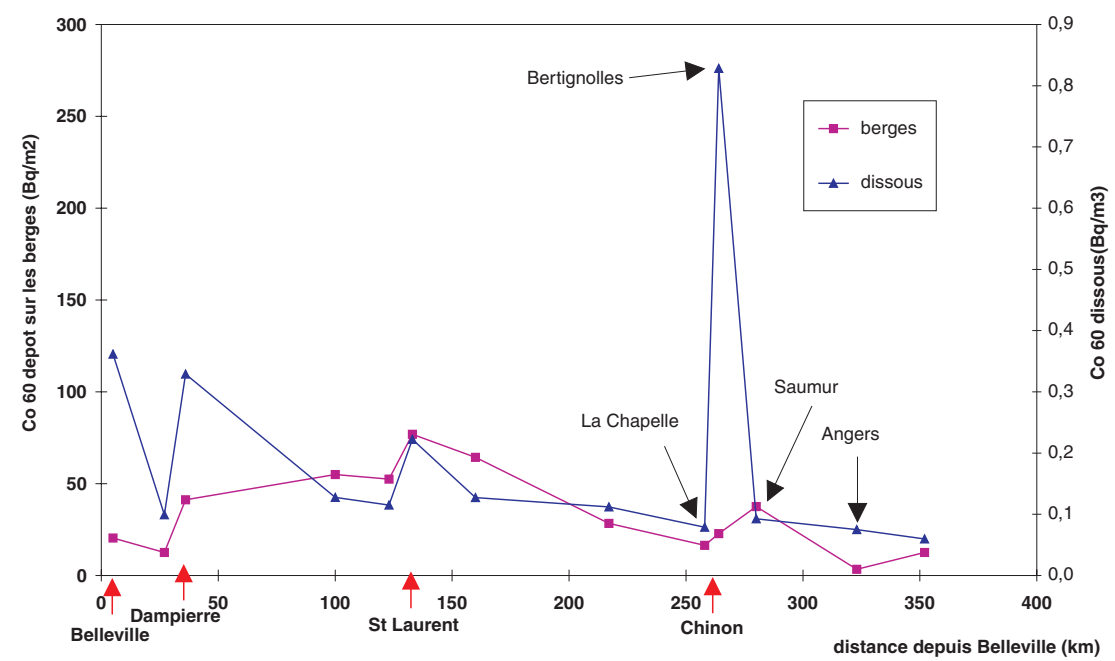

Fig. 11. - Evolution le long de la Loire des concentrations moyennes en ${ }^{60} \mathrm{Co}$ dans le cours d'eau et sur les berges (moyennes sur la période de 1994 à 1999).

Fig. 11. - Spatial distribution of ${ }^{60} \mathrm{Co}$ in dissolved fraction and in shore deposits (1994 to 1999 average values). 
soudaine des sédiments accumulés dans cette retenue. Cependant, l'activité spécifique des sédiments remis en mouvement, exprimée en $\mathrm{Bq} / \mathrm{kg}$, est inférieure à celle des MES lors du passage d'un rejet radioactif (Fig. 12) et ne peut donc donner lieu à une augmentation de l'activité spécifique des sédiments déposés en aval.

\subsection{Evaluation de la dose au public liée aux rejets de ces centrales}

\subsubsection{Le modèle CALVADOS}

CALVADOS est destiné à évaluer les transferts dans l'environnement et la dose à l'homme, liée aux rejets de radionucléides dans les cours d'eau. A partir des résultats de CRESCENDO, pour une station donnée, il simule, avec un pas de temps mensuel, d'une part les transferts dans les cours d'eau jusqu'aux poissons, d'autre part les transferts aux sols et aux productions agricoles via l'irrigation et l'abreuvage des animaux. II calcule in fine les doses annuelles à l'homme à partir des voies d'exposition suivantes:

- l'ingestion d'eau, de poisson, de végétaux ou de produits animaux contaminés (exposition interne) ;

- l'inhalation de poussières composées de particules de sol contaminé en suspension (exposition interne) ;

- l'exposition externe due au séjour sur un sol contaminé, ou au séjour

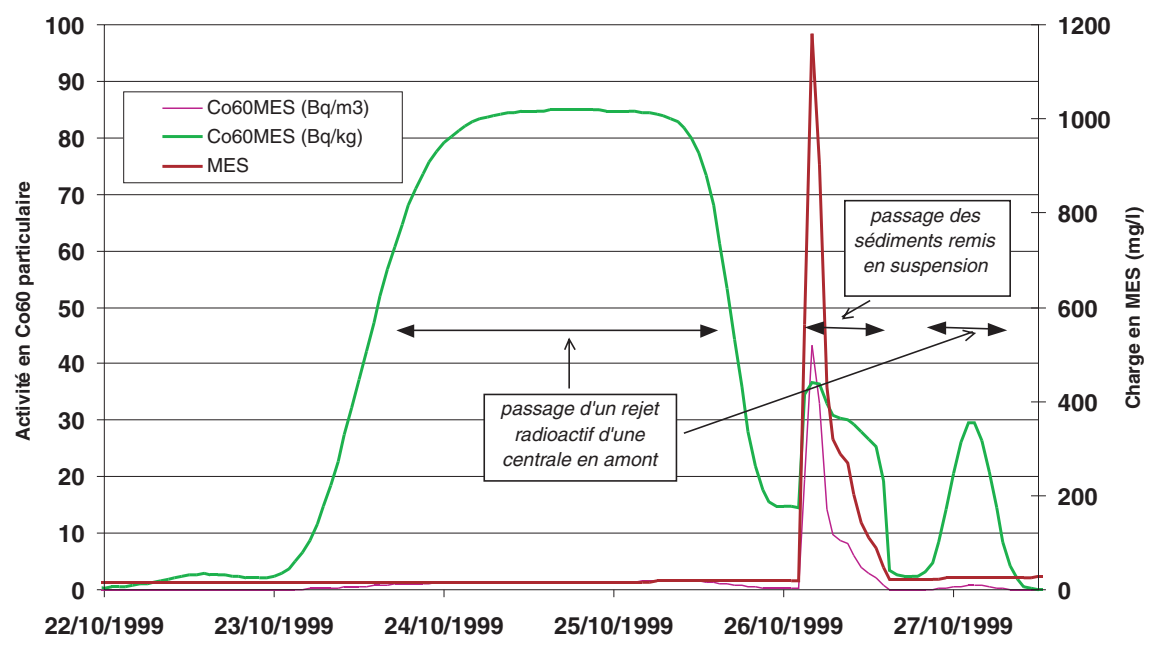

Fig. 12. - Evolution de la concentration en MES et en cobalt 60 particulaire en aval du barrage de Blois lors de l'épisode de remise en suspension du 26 octobre 1999.

Fig. 12. - Evolution of suspended particulate matter and particulate ${ }^{60} \mathrm{Co}$ concentrations downstream of the Blois dam, during the resuspension event of Oct 26, 1999. 
sur les berges de la rivière où sont en particulier présents des sédiments contaminés, ou lors de baignades et d'activités nautiques.

Ce modèle tient compte de la variabilité saisonnière des régimes du fleuve, des pratiques agricoles et des habitudes humaines.

Le principe du modèle consiste à considérer les flux de masse entre les différentes composantes de l'écosystème simplifié. A chaque pas de temps, le stock de radionucléides dans un compartiment est égal au stock au pas de temps précédent, auquel s'ajoutent les apports provenant d'autres compartiments et les pertes dues soit à la migration des radionucléides vers d'autres compartiments, soit à la décroissance radioactive.

Des modélisations différentes sont utilisées pour le tritium et le carbone 14 , d'une part, et les autres radionucléides rejetés par les installations, d'autre part.

Les valeurs des paramètres propres aux sites ont été évaluées d'après une étude des pratiques agricoles dans la zone concernée. Les descripteurs des habitudes de vie ont été tirés de la littérature existante. Les valeurs concernant le régime alimentaire, qui proviennent d'études effectuées par l'INSEE et le CEA, correspondent à la moyenne nationale pour les adultes (Tableau 1). Celles relatives au mode de vie ont été plus difficiles à obtenir. II a fallu, par exemple, évaluer le temps consacré à des activités de loisirs pratiquées au bord du fleuve (pêche, marche, etc.). En l'absence de références bi- bliographiques ou de données propres au site, une valeur de $10 \%$ du temps tout au long de l'année a été retenue de façon assez prudente.

\subsubsection{Evaluations des doses} annuelles moyennes le long de la Loire et de la Vienne

Parmi les radionucléides émis par les centrales, les plus fortes contributions à la dose reçue (Tableau 2) peuvent être attribuées au tritium par l'ingestion d'eau, au ${ }^{14} \mathrm{C}$ par la consommation de poissons provenant du fleuve, et aux éléments ${ }^{110 \mathrm{~m}} \mathrm{Ag}$ et ${ }^{60} \mathrm{Co}$ par le biais des activités de loisirs sur les rives, avec une dose annuelle, pour chacune de ces voies, d'environ 0,1 microsievert. Les voies de contamination agricoles (par l'irrigation des cultures et l'eau bue par le bétail) n'apportent qu'une plus faible contribution à la dose totale.

Tous radionucléides confondus, la voie d'exposition dominante est due dans une station comme Blois, à la fréquentation des rives du fleuve dans des zones de dépôt de sédiment fins. Les autres voies d'exposition externe (baignades, activités nautiques, activités agricoles sur sol irrigué) sont inférieures de deux à trois ordres de grandeur à celle provenant des sédiments des berges (Fig. 13).

L'exposition externe due aux rejets radioactifs reste toutefois très faible (0,037 microsievert par an) comparée à celle imputable aux radionucléides naturels $\left({ }^{40} \mathrm{~K},{ }^{226} \mathrm{Ra}\right.$ et $\left.{ }^{232} \mathrm{Th}\right)$ présents 
Tableau 1 - Ration alimentaire et mode de vie pris en compte dans le calcul de doses.

Table 1 - Diet values and usage factors selected for dose calculation.

\begin{tabular}{|c|c|c|c|c|c|}
\hline $\begin{array}{c}\text { Type } \\
\text { de produits }\end{array}$ & Produit & $\begin{array}{l}\text { Ration } \\
\text { (g frais } \\
\text { par jour) }\end{array}$ & Type d'exposition & $\begin{array}{l}\text { Temps } \\
\text { passé } \\
\%\end{array}$ & $\begin{array}{c}\text { Période } \\
\text { d'exposition }\end{array}$ \\
\hline Boissons & Eau & 1200 & \multirow{4}{*}{$\begin{array}{c}\text { pour un agriculteur } \\
\text { exposition au sol irrigué } \\
\text { (irradiation externe et } \\
\text { inhalation) }\end{array}$} & \multirow[t]{4}{*}{15} & \multirow{4}{*}{$\begin{array}{l}\text { toute } \\
\text { l'année }\end{array}$} \\
\hline \multirow{7}{*}{$\begin{array}{l}\text { Produits } \\
\text { végétaux }\end{array}$} & Légumes racines & & & & \\
\hline & pomme de terre & 240 & & & \\
\hline & Légumes fruits & & & & \\
\hline & haricots & 90 & \multirow[t]{2}{*}{ baignade } & \multirow[t]{2}{*}{10} & \multirow[t]{2}{*}{ juil à sept } \\
\hline & fruits & 195 & & & \\
\hline & Légumes feuilles & & & & \\
\hline & salade & 40 & activités nautiques & 10 & juil à sept \\
\hline Produits laitiers & Lait (UHT + cru) & 260 & \multirow{3}{*}{$\begin{array}{l}\text { exposition sur les berges } \\
\text { (pêche, promenade) }\end{array}$} & \multirow[t]{3}{*}{10} & \multirow{3}{*}{$\begin{array}{l}\text { toute } \\
\text { l'année }\end{array}$} \\
\hline \multirow{2}{*}{$\begin{array}{l}\text { Viande } \\
\text { et poisson }\end{array}$} & Viande (bœuf) & 120 & & & \\
\hline & Poisson & 30 & & & \\
\hline
\end{tabular}

Tableau 2 - Principaux radionucléides contribuant à la dose individuelle à Blois (moyenne 19941999).

Table 2 - Contribution of major radionuclides to individual dose in Blois (1994 to 1999 average)

\begin{tabular}{|c|c|c|c|c|c|c|}
\hline Dose (Sv/an) & Co 60 & Ag 110m & Cs 137 & C14 & H 3 & Total \\
\hline sédiments berges & 9.4E-08 & $1.5 \mathrm{E}-07$ & 3.2E-09 & - & - & $3.6 \mathrm{E}-07$ \\
\hline eau de boisson & $1.9 \mathrm{E}-10$ & $1.4 \mathrm{E}-10$ & $5.8 \mathrm{E}-10$ & 3.0E-09 & 1.1E-07 & $1.2 \mathrm{E}-07$ \\
\hline poissons & 1.3E-09 & 1.7E-11 & $2.8 \mathrm{E}-08$ & $1.9 \mathrm{E}-07$ & $2.5 \mathrm{E}-09$ & $2.5 \mathrm{E}-07$ \\
\hline végétaux irrigués & 1.0E-09 & 1.2E-09 & 7.8E-09 & 5.2E-09 & $6.2 \mathrm{E}-08$ & $8.9 \mathrm{E}-08$ \\
\hline lait & $1.4 \mathrm{E}-11$ & $1.1 \mathrm{E}-10$ & $1.2 \mathrm{E}-10$ & 4.3E-10 & $1.9 \mathrm{E}-08$ & $2.0 \mathrm{E}-08$ \\
\hline viande & 5.3E-11 & $1.4 \mathrm{E}-11$ & $1.7 \mathrm{E}-10$ & $3.6 \mathrm{E}-10$ & $6.2 \mathrm{E}-09$ & $8.4 \mathrm{E}-09$ \\
\hline Total & $9.8 \mathrm{E}-08$ & 1.6E-07 & 4.0E-08 & 2.0E-07 & $2.1 \mathrm{E}-07$ & 8.6E-07 \\
\hline
\end{tabular}

sur les rives du fleuve, laquelle s'élève à 50 microsieverts par an.

Les doses à l'homme dues à l'ensemble des radionucléides liquides rejetées par les centrales varient de 0,15 à 1,7 microsieverts par an en fonction des stations. Les différences entre stations (Fig. 14) sont liées d'une part à la dilution incomplète des effluents dans les zones proches des rejets par rapport aux zones plus éloi- gnées (différence entre Nouan et Blois par exemple), d'autre part à l'hétérogénéité spatiale de la sédimentation sur les berges (différence entre Saumur et Angers, par exemple). II convient cependant de nuancer ces résultats en rappelant que l'on suppose, de façon très prudente, qu'il y a consommation d'eau de boisson provenant du fleuve, même dans la zone proche du rejet, 


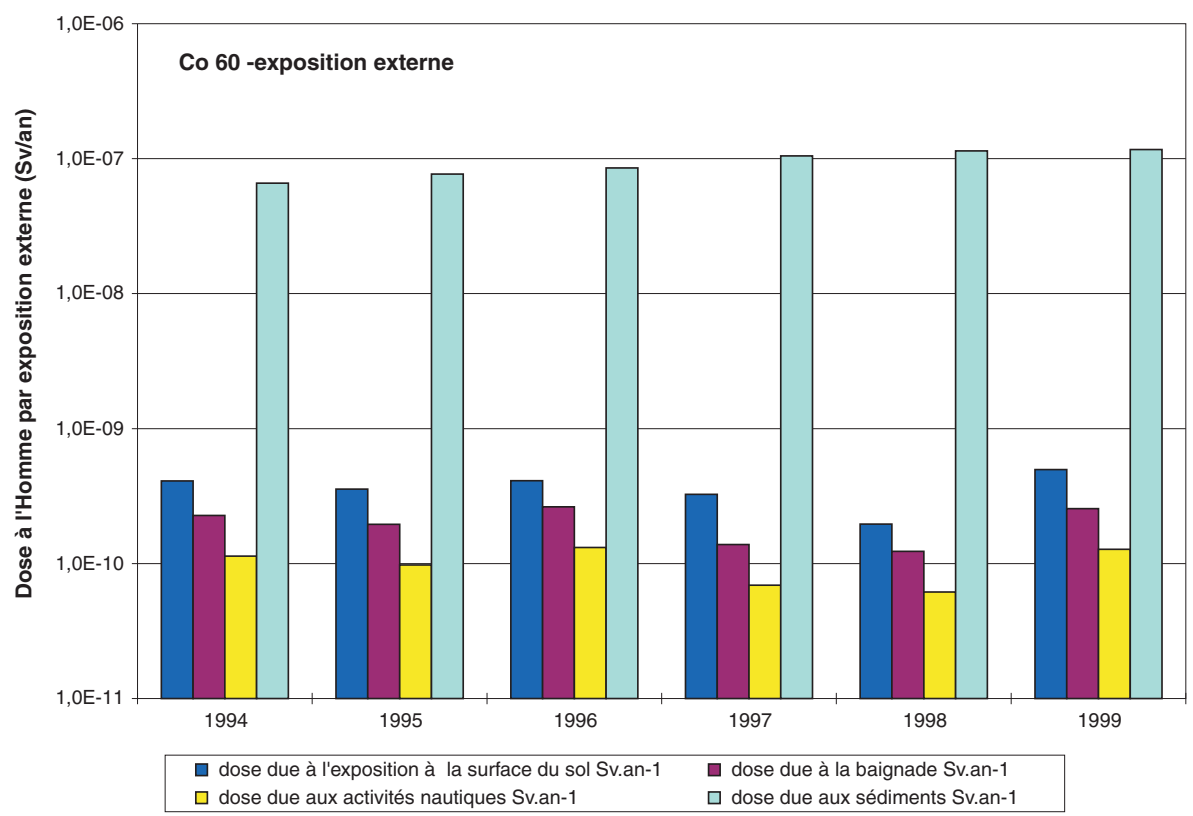

Fig. 13. - Estimation des doses par irradiation externe à Blois.

Fig. 13. - Estimation of external exposure in Blois.

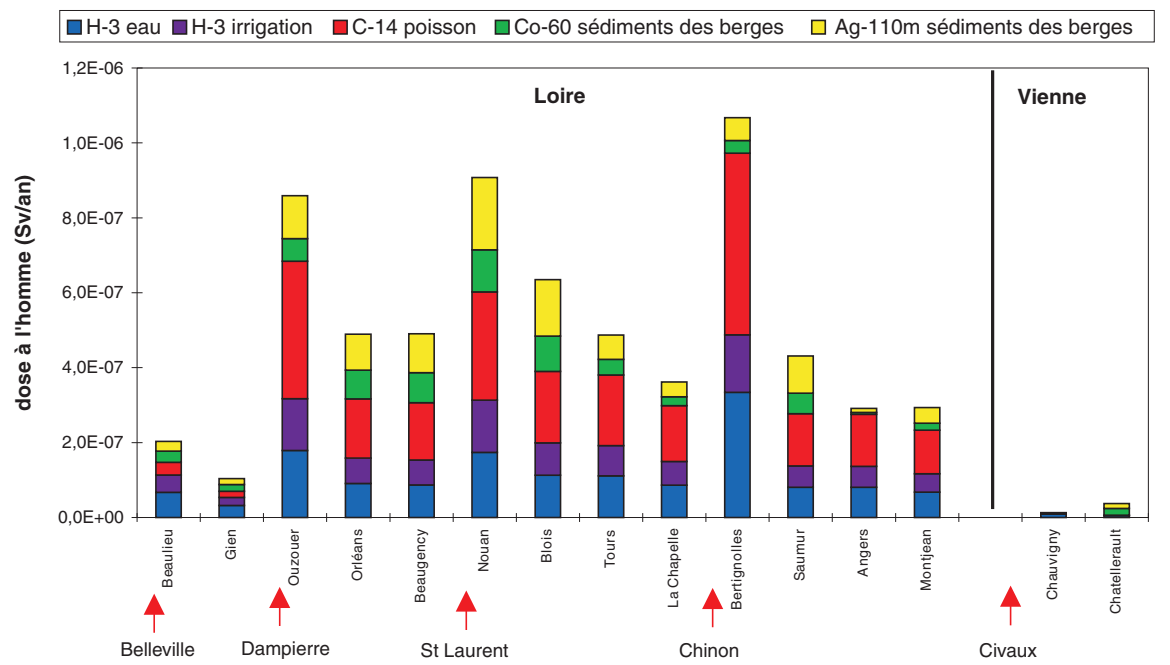

Fig. 14. - Evaluation de la dose moyenne liée aux rejets liquides des CNPE de la Loire et de la Vienne (les flèches représentent les rejets des centrales).

Fig. 14. - Evaluation of average dose due to liquid releases of NPP on the Loire and Vienne rivers (arrows represent power plants release). 
et que le temps passé sur les berges du fleuve a été volontairement fixé à une valeur majorante.

\section{L'ESTUAIRE DE LA LOIRE}

Le peu de données existantes sur la radioactivité de l'estuaire de la Loire a motivé, dans le cadre du projet Radioécologie Loire, un effort important d'acquisition de mesures visant à caractériser les niveaux de radioactivité dans la masse d'eau et les sédiments. Ces mesures constituent une base de connaissances essentielle pour :

- identifier les paramètres déterminants dans le comportement des radionucléides en estuaire (salinité, teneur en matière organique...) et vérifier la cohérence avec les résultats d'expérimentation en laboratoire menée par le CEREGE ;

- fournir les échelles de variation des phénomènes à modéliser : influence de la marée, des cycles de marée, des débits fluviaux et des saisons ;

- caler et valider les modèles numériques.

\subsection{Etudes saisonnières de la radioactivité dans le bouchon vaseux}

Des prélèvements de sédiments de fond, de l'eau et des matières en suspension ont été réalisés dans l'estuaire à 8 dates différentes, entre juillet 1998 et septembre 1999 (Photo 4).
Ces dates ont été choisies de façon à inclure différentes conditions de marée, depuis celles de morte-eau jusqu'à celles de vive-eau, et ce pour différents débits du fleuve (Tableau 3).

Les échantillons ont été prélevés à différentes profondeurs de la colonne d'eau (surface, intermédiaire et fond). 28 échantillons d'eau de grand volume (300 à 2000 litres) ont été prélevés, ainsi que 12 échantillons de crème de vase et 11 de sédiments de fond (Fig. 15). De plus, pour tenir lieu de référence marine, un échantillon d'eau de mer a été prélevé le long de la cote Atlantique, en dehors de la zone soumise à l'influence de l'estuaire, à hauteur du Croisic.

Les analyses portaient sur le ${ }^{14} \mathrm{C}$ (dissous et associé aux particules de matières organiques), le ${ }^{90} \mathrm{Sr}$ (dissous et particulaire), le ${ }^{3} \mathrm{H}$ (sous forme d'eau tritiée ou de tritium lié à la matière organique), les émetteurs gamma naturellement présents (chaînes de désintégration de l'uranium et du thorium, ${ }^{7} \mathrm{Be}$ et ${ }^{40} \mathrm{~K}$ ), et les émetteurs gamma artificiels (principalement les isotopes du cobalt et du césium) dans les fractions dissoutes et particulaires.

De plus, afin d'explorer l'importance de l'accumulation de radionucléides dans ce secteur, 8 carottes de sédiments ont été prélevées dans les zones de dépôt (berges de l'estuaire, bras secondaires, vasières latérales), et deux autres sur le littoral océanique, à proximité de l'embouchure, à Saint-Brévin (Fig. 16). 


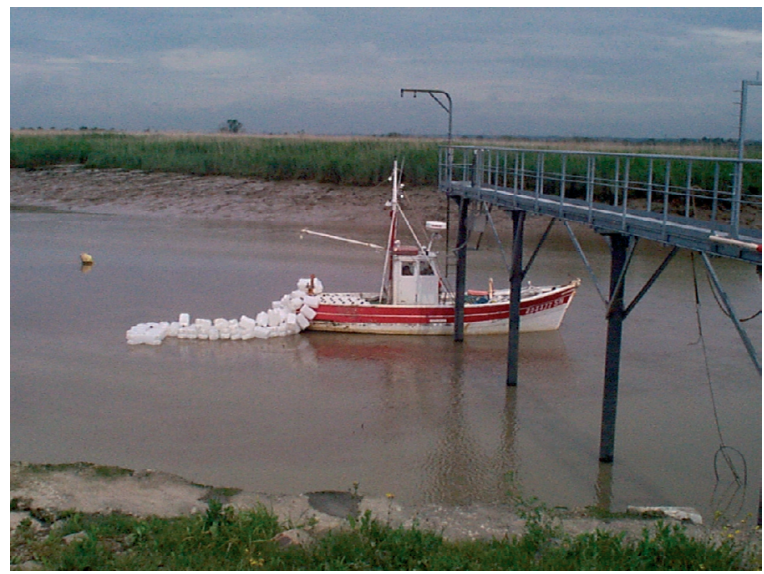

Photo 4. - Bateau de prélèvement "Anne de Bretagne » à la sortie du bras de Cordemais. Le chapelet de bidons de 30 litres est destiné à recueillir l'eau prélevée et à la transporter jusqu'à la centrifugeuse située sur la rive.

Photo 4. - The Anne de Bretagne ship at the outlet of the Cordemais channel. The string of 30 liters tanks is used to collect the sampled water and transport it to the centrifuge on shore.

Tableau 3. - Programme d'échantillonnage dans l'estuaire de la Loire.

Table 3. - Sampling program in the Loire estuary.

\begin{tabular}{|c|c|c|c|c|c|}
\hline $\begin{array}{l}\text { Numéro } \\
\text { de cam- } \\
\text { pagne }\end{array}$ & Date & $\begin{array}{l}\text { Coeffi- } \\
\text { cient de } \\
\text { marée }\end{array}$ & $\begin{array}{l}\text { Débit de la } \\
\text { Loire } \\
\left(\mathrm{m}^{3} . \mathrm{s}^{-1}\right)\end{array}$ & $\begin{array}{l}\text { Position du } \\
\text { maximum } \\
\text { de turbidité }\end{array}$ & Type d'échantillon \\
\hline 1 & 03/07/1998 & 41 & 292 & Cordemais & $\begin{array}{l}\text { profil vertical à marée } \\
\text { haute }\end{array}$ \\
\hline 2 & 08/07/1998 & 71 & 270 & Cordemais & $\begin{array}{l}\text { variabilité sur un cycle de } \\
\text { marée : profil vertical à } \\
\text { marée haute, au jusant, à } \\
\text { marée basse, au flot et à } \\
\text { la marée haute suivante }\end{array}$ \\
\hline 3 & $15 / 09 / 1998$ & 47 & 225 & Le Pellerin & $\begin{array}{l}\text { profil vertical à marée } \\
\text { haute }\end{array}$ \\
\hline 4 & 05/02/1999 & 78 & 1310 & Donges & $\begin{array}{l}\text { variabilité sur un cycle de } \\
\text { marée : profil vertical à } \\
\text { marée haute, au jusant, à } \\
\text { marée basse, au flot et à } \\
\text { la marée haute suivante }\end{array}$ \\
\hline 5 & $25 / 02 / 1999$ & 51 & 3070 & St-Nazaire & Transect au jusant \\
\hline 6 & $17 / 05 / 1999$ & 107 & 663 & Cordemais & profil vertical à mi-flot \\
\hline 7 & $12 / 07 / 1999$ & 93 & 267 & Le Pellerin & profil vertical à mi-flot \\
\hline 8 & $28 / 09 / 1999$ & 101 & 354 & Le Pellerin & profil vertical à mi-flot \\
\hline Mer & $16 / 09 / 1998$ & 55 & & & $\begin{array}{l}\text { prélèvement en surface } \\
\text { pendant } 16 \mathrm{~h} \text { à partir de } \\
\text { pleine mer dans la rade } \\
\text { du Croisic }\end{array}$ \\
\hline
\end{tabular}




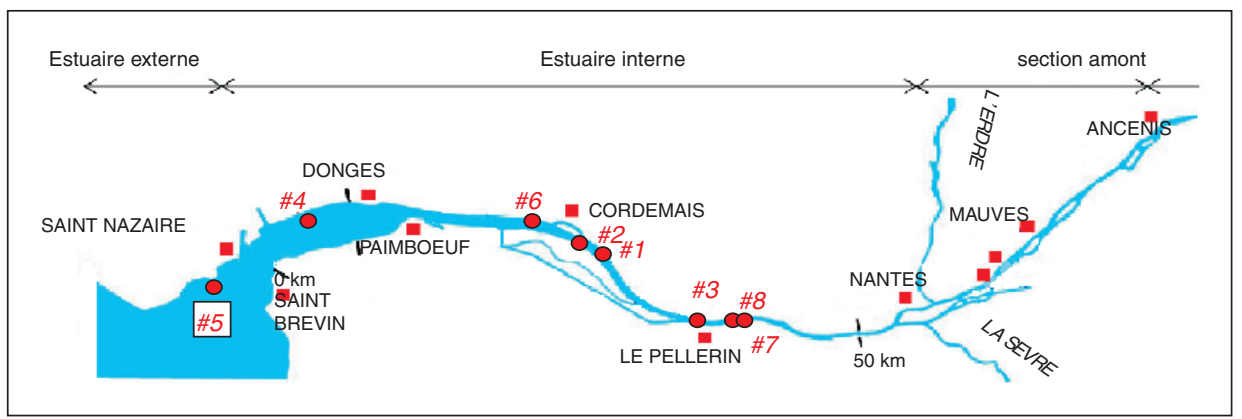

- stations d'échantillonnage de l'eau et des sédiments

Fig. 15. - Localisation des points de prélèvements dans le bouchon vaseux. Les nombres représentent les numéros de campagnes (voir Tableau 3).

Fig. 15. - Map of sampling sites in the turbidity maximum. Numbers represent survey numbers (see Table 3).

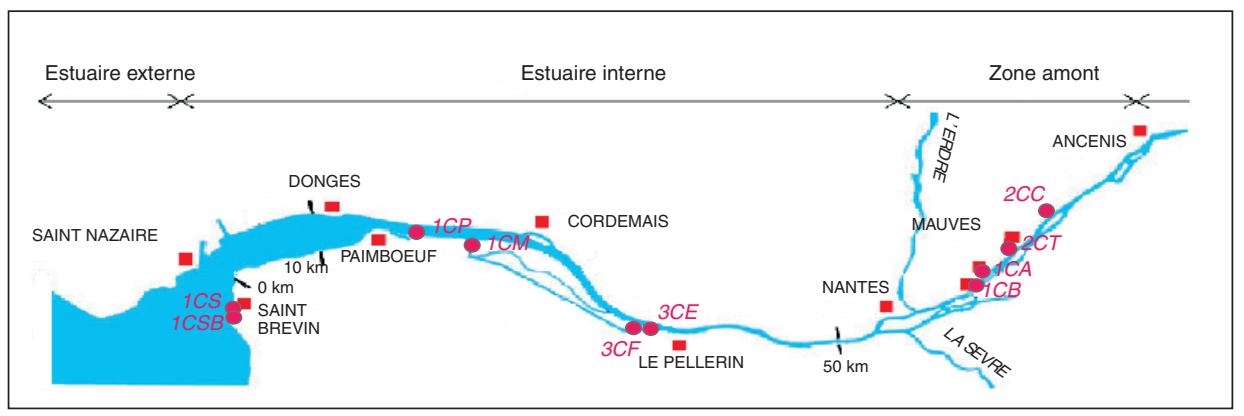

- Prélèvements de carottes sédimentaires

Fig. 16. - Localisation des points de carottages.

Fig. 16. - Map of sediment core sampling sites

Les résultats sont présentés de façon plus détaillée en [1] pour le ${ }^{137} \mathrm{Cs}$ et le ${ }^{90} \mathrm{Sr}$, en [2] pour le ${ }^{14} \mathrm{C}$ et en [3] pour les carottes de sédiments.

\subsection{Radioactivité liée aux particules}

La radioactivité naturelle relevée dans les MES et dans les sédiments est dominée par le ${ }^{40} \mathrm{~K}$ (plus de $600 \mathrm{~Bq} / \mathrm{kg}$ ), suivi du ${ }^{210} \mathrm{~Pb}(150 \mathrm{~Bq} / \mathrm{kg})$, des familles ${ }^{238} \mathrm{U}$ et ${ }^{232} \mathrm{Th}(50 \mathrm{~Bq} / \mathrm{kg})$ et $\mathrm{du}{ }^{7} \mathrm{Be}(30 \mathrm{~Bq} / \mathrm{kg})$.

Les concentrations de radionucléides d'origine anthropique vont de seulement $10 \mathrm{~Bq} / \mathrm{kg}$ pour le ${ }^{137} \mathrm{Cs}$ à des valeurs inférieures à la limite de détection $(0,1 \mathrm{~Bq} / \mathrm{kg})$, dans l'ordre d'abondance suivant: ${ }^{137} \mathrm{Cs}^{14} \mathrm{COBT}$ (tritium organiquement lié) ${ }^{60} \mathrm{Co}{ }^{134} \mathrm{Cs}$, ${ }^{110 \mathrm{~m}} \mathrm{Ag},{ }^{90} \mathrm{Sr}$.

Les radionucléides comme le ${ }^{58} \mathrm{Co}$, le ${ }^{54} \mathrm{Mn}$, le ${ }^{131} \mathrm{l}$, bien que présents dans les rejets des centrales, sont toujours 
restés inférieurs à la limite de détection dans les particules estuariennes.

Ces résultats de mesures ont été comparés aux concentrations dans la Loire calculées avec le modèle CRESCENDO pour les radionucléides rejetés par les centrales (cf. II supra). Ce sont les résultats de calcul à Montjean-sur-Loire (Fig. 1), situé à la limite de l'incursion des marées, qui ont servi à cette comparaison.

Les concentrations observées de ${ }^{60} \mathrm{Co}$ et de ${ }^{110 m} \mathrm{Ag}$ se trouvent dans l'ordre de grandeur des contributions calculées de la Loire (Fig. 17), tandis que celles de ${ }^{137} \mathrm{C}$ s et ${ }^{90} \mathrm{Sr}$ sont respectivement 20 et 100 fois supérieures aux valeurs calculées pour les rejets industriels, démontrant que les retombées des tirs nucléaires (en ${ }^{137} \mathrm{Cs}$ et ${ }^{90} \mathrm{Sr}$ ) et de l'accident de Tchernobyl $\left({ }^{137} \mathrm{Cs}\right)$ restent les principales sources de ces radionucléides.

Les concentrations en tritium lié à la matière organique (OBT) exprimées en Bq/l d'eau de combustion dépassent lors de chaque campagne la moyenne de l'activité en tritium dissous, HTO (Fig. 18). La moyenne de la teneur en OBT est de $20,55 \mathrm{~Bq} / \mathrm{L}$ d'eau de combustion avec un écart- type de 5,7Bq/L. L'hétérogénéité à une date donnée entre les échantillons de surface et la crème de vase est importante sans que l'on puisse dégager de tendance claire de répartition le long de la verticale. Ces concentrations sont cependant du même ordre de grandeur que celles mesurées dans les sédiments de Loire (Tableau 4). Elles ne semblent pas être influencées par les rejets liquides des centrales nucléaires, comme le montre la concentration mesurée à Belleville, en amont de toutes les installations. La détermination de l'origine biogéochimique de la matière organique, à partir de marqueurs lipidiques [2], montre que la composante dominante est d'origine terrigène (végétaux terrestres) ce qui conforte la thèse de l'absence de marquage par les rejets liquides des centrales. L'enrichissement isotopique par rapport à HTO peut résulter d'une part d'un fractionnement isotopique lors de la dégradation de la matière organique qui aboutirait à un enrichissement progressif des composés organiques particulaires, d'autre part d'une formation de la matière organique à une époque plus ancienne

Tableau 4. - Teneurs en tritium lié à la matière organique mesurées en Loire.

Table 4. - Organically bound tritium measurements in the Loire river.

\begin{tabular}{|c|c|c|c|}
\hline $\begin{array}{c}\text { Localisation } \\
\text { du prélèvement }\end{array}$ & $\begin{array}{c}\text { Nature de l'échantillon } \\
\text { et année de prélèvement }\end{array}$ & OBT en Bq/L & Référence EDF \\
\hline amont Belleville & MES 1999 & 12 à 13 & [atelier C-14] \\
\hline amont Belleville & sédiment 1998 & $31,5 \pm 0,9$ & [décennale BEL] \\
\hline Orléans à Blois & sédiment 1993 & 12 à 36 & [décennale SLA] \\
\hline Gien à Orléans & sédiment 1992 & 20 à 29 & [décennale DAM] \\
\hline
\end{tabular}


- surface $\square$ mi profondeur $\square$ crème de vase $\circ$ fond $*$ Loire (apport industriel)
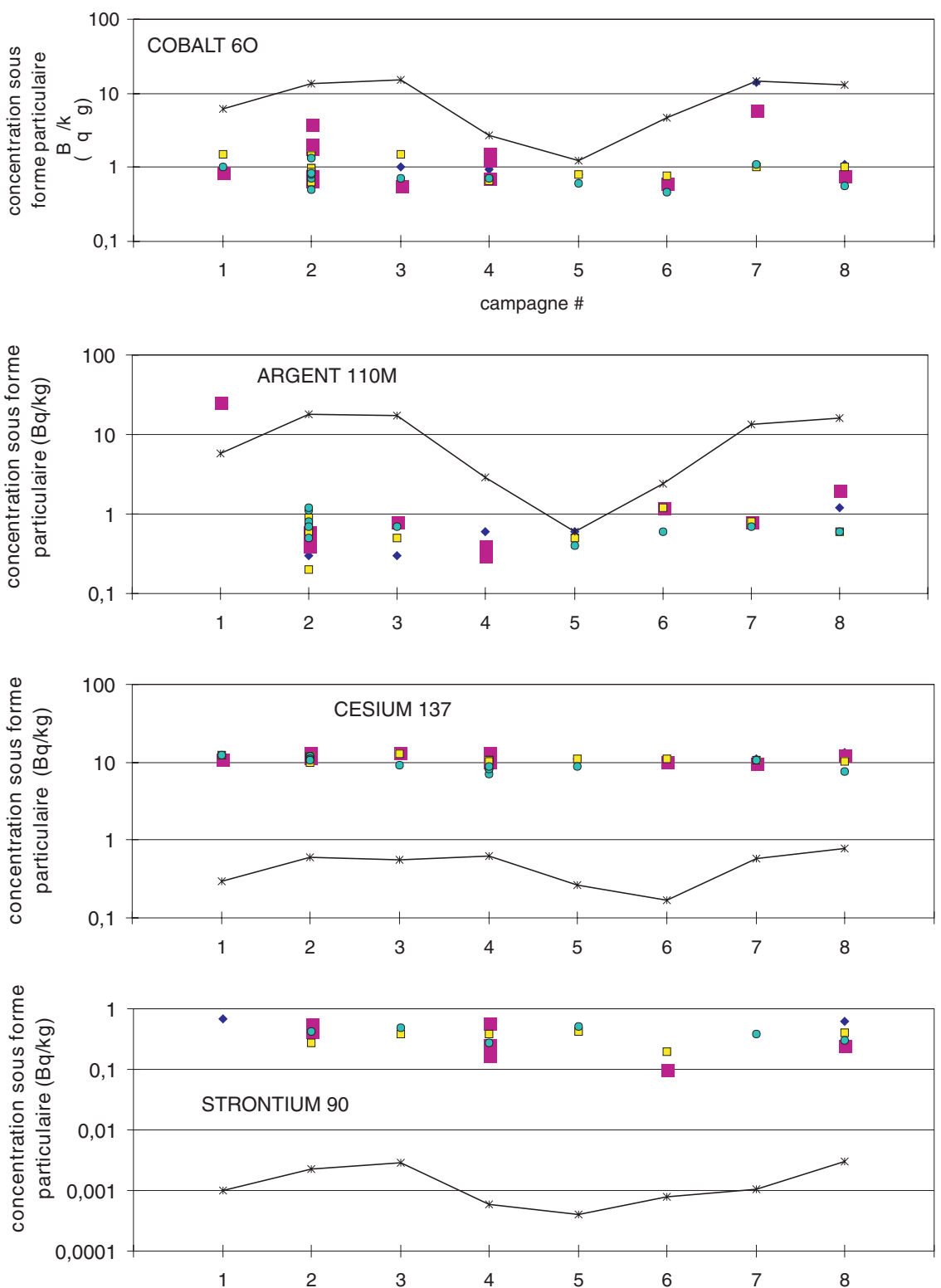

Fig. 17. - Comparaison entre les concentrations observées dans le bouchon vaseux et les apports calculés en Loire à partir des rejets industriels.

Fig. 17. - Comparison between observed concentrations in the turbidity maximum and Loire calculated input from industrial releases. 

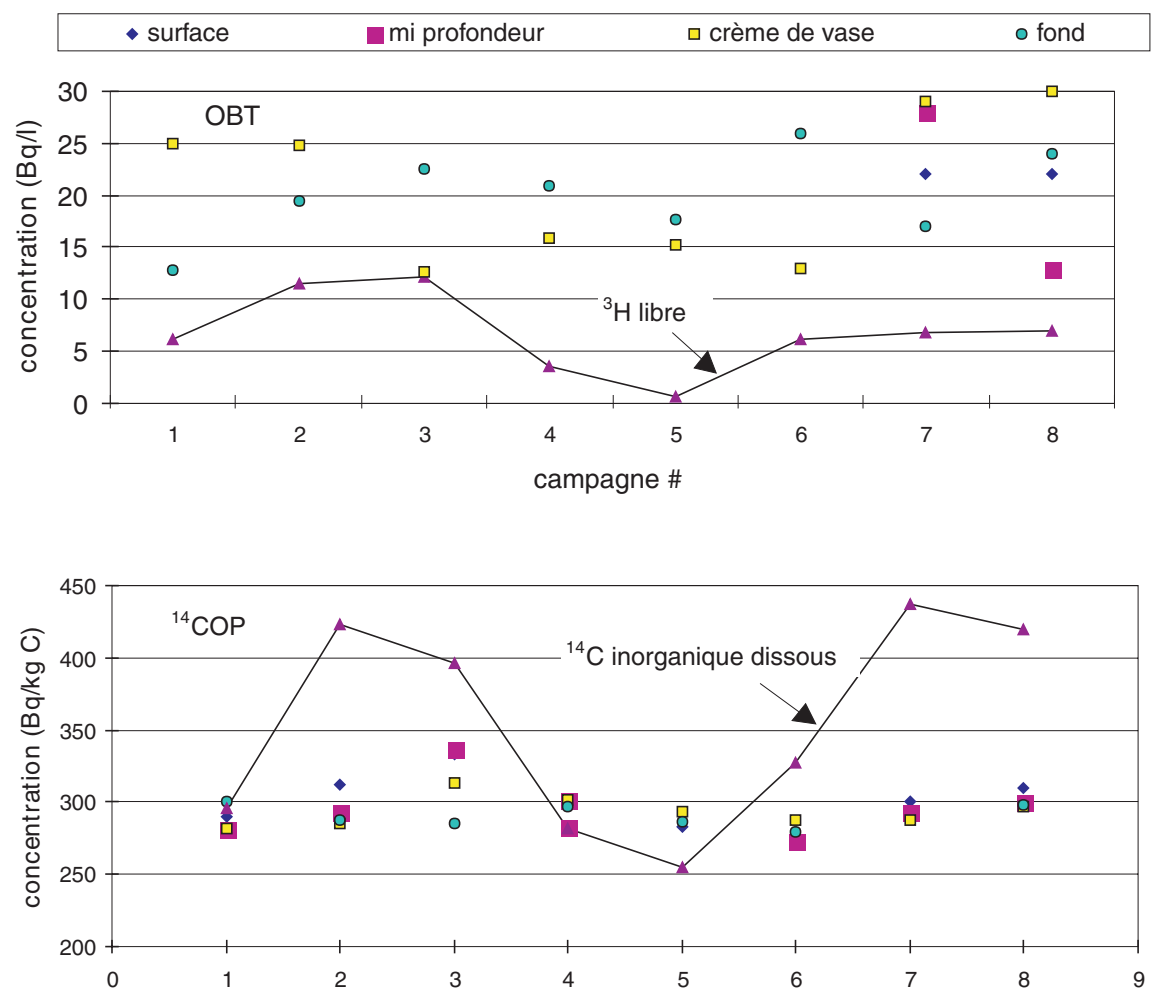

Fig. 18. - Concentrations en tritium $(\mathrm{OBT})$ et en carbone $14\left({ }^{14} \mathrm{COP}\right)$ associés à la matière organique particulaire. Comparaison avec les formes minérales dissoutes (eau tritiée et $14 \mathrm{C}$ inorganique dissous).

Fig. 18. - Tritium (OBT) and carbone $14\left(\mathrm{PO}^{14} \mathrm{C}\right)$ concentration in particulate organic matter. Comparison with dissolved inorganic forms (tritiated water and dissolved inorganic ${ }^{14} \mathrm{C}$ ).

lorsque les concentrations ambiantes en HTO étaient plus élevées, comme le confirme les teneurs en ${ }^{14} \mathrm{C}$.

Les concentrations en ${ }^{14} \mathrm{C}$ organique particulaire $\left({ }^{14} \mathrm{COP}\right)$ sont relativement stables au cours des 8 campagnes, avec une moyenne de $296 \mathrm{~Bq} / \mathrm{kg}$ de carbone et un écarttype de $15 \mathrm{~Bq} / \mathrm{kg}$ de carbone (Fig. 18). Elles sont significativement plus basses que les concentrations observées dans la phase minérale dissoute (348 Bq/kg de carbone). Les valeurs de delta ${ }^{13} \mathrm{C}$ (différence entre le rapport ${ }^{13} \mathrm{C} /{ }^{12} \mathrm{C}$ de l'échantillon et celui de la Pee Dee Belemnite, fossile calcaire de référence ; cette grandeur permet d'évaluer le fractionnement isotopique entre les isotopes du carbone stable ${ }^{13} \mathrm{C}$ et ${ }^{12} \mathrm{C}$; elle est sans unité, mais il est d'usage de l'exprimer en pour mille, en la multipliant 
par 1000) de la matière organique, entre -25 et -27 pour mille, confirment l'origine terrestre de ce matériau, déjà mise en évidence par la composition lipidique. On peut donc considérer que le carbone 14 rejeté dans les cours d'eau par les centrales, même s'il participe à la production algale et microbienne, est rapidement dégradé dans l'estuaire où ne subsistent que les substances organiques résistant à la décomposition bactérienne. II en résulte que le marquage de la phase particulaire a pour origines, d'une part la production naturelle de ${ }^{14} \mathrm{C}$, et d'autre part les retombées atmosphériques sur les bassins versants.

Les concentrations en ${ }^{14} \mathrm{C}$ particulaire, plus fortes que les concentrations actuelles du $\mathrm{CO}_{2}$ atmosphérique (250 Bq/kg de carbone), traduisent l'âge plus ancien de la matière organique d'origine pédologique, dont le carbone a été incorporé par la photosynthèse, il y a plusieurs dizaines d'années, lorsque le marquage de l'atmosphère par les retombées des essais nucléaires était plus important (jusqu'à $450 \mathrm{~Bq} / \mathrm{kg}$ de carbone au début des années soixante). Ce marquage ancien de la matière organique particulaire des estuaires avait déjà été mis en évidence par des mesures d'activité en ${ }^{14} \mathrm{C}$ dans la Gironde au début des années 1980 [7].

\subsection{Radioactivité dissoute}

Le tritium est le plus abondant des radionucléides observés, avec une concentration moyenne de $7,6 \mathrm{~Bq} / \mathrm{l}$, suivi de près par le ${ }^{40} \mathrm{~K}(2,7 \mathrm{~Bq} / \mathrm{l})$. Le ${ }^{14} \mathrm{C}$, le ${ }^{90} \mathrm{Sr}$ et le ${ }^{7} \mathrm{Be}$ sont inférieurs de trois ordres de grandeur, allant de 3 à $5 \mathrm{mBq} / \mathrm{l}$. Vient ensuite le ${ }^{137} \mathrm{Cs}(0,4$ $\mathrm{mBq} / \mathrm{l})$. Le ${ }^{131} \mathrm{I}$ et le ${ }^{60} \mathrm{Co}$ n'ont été détectés que dans de rares échantillons (moyennes de 0,05 et 0,01 mBq/l, respectivement).

Les variations des concentrations de tritium observées coïncident avec les calculs (Fig. 19). La concordance est moins bonne pour le ${ }^{14} \mathrm{C}$ : l'amplitude des variations dans l'estuaire est inférieure à celle issue des calculs dans le fleuve, ce qui peut être imputé à une surestimation des apports industriels en ${ }^{14} \mathrm{C}$, élément que le modèle CRESCENDO considère comme un traceur dissous conservatif. De plus, afin de permettre la comparaison avec les mesures, l'activité volumique $\left(\mathrm{Bq} / \mathrm{m}^{3}\right)$ calculée par le modèle est transformée en activité spécifique (Bq/kg de carbone stable), en supposant que la concentration en carbone inorganique dissous apportée par la Loire est constante et égale à 20 $\mathrm{mg} / \mathrm{l}$. Enfin les valeurs calculées en Loire ne tiennent pas compte des sources naturelles de ${ }^{14} \mathrm{C}$ dont la concentration moyenne est de 250 $\mathrm{Bq} / \mathrm{kg}$ ce qui explique le niveau de base relativement élevé observé dans l'estuaire.

Les concentrations de ${ }^{137} \mathrm{Cs}$ dissous augmentent de façon linéaire avec la salinité (Fig. 20), mettant en évidence un apport de ${ }^{137} \mathrm{Cs}$ d'origine océanique, plutôt qu'une désorption liée à l'augmentation de salinité. D'ailleurs, les concentrations en ${ }^{137} \mathrm{Cs}$ 

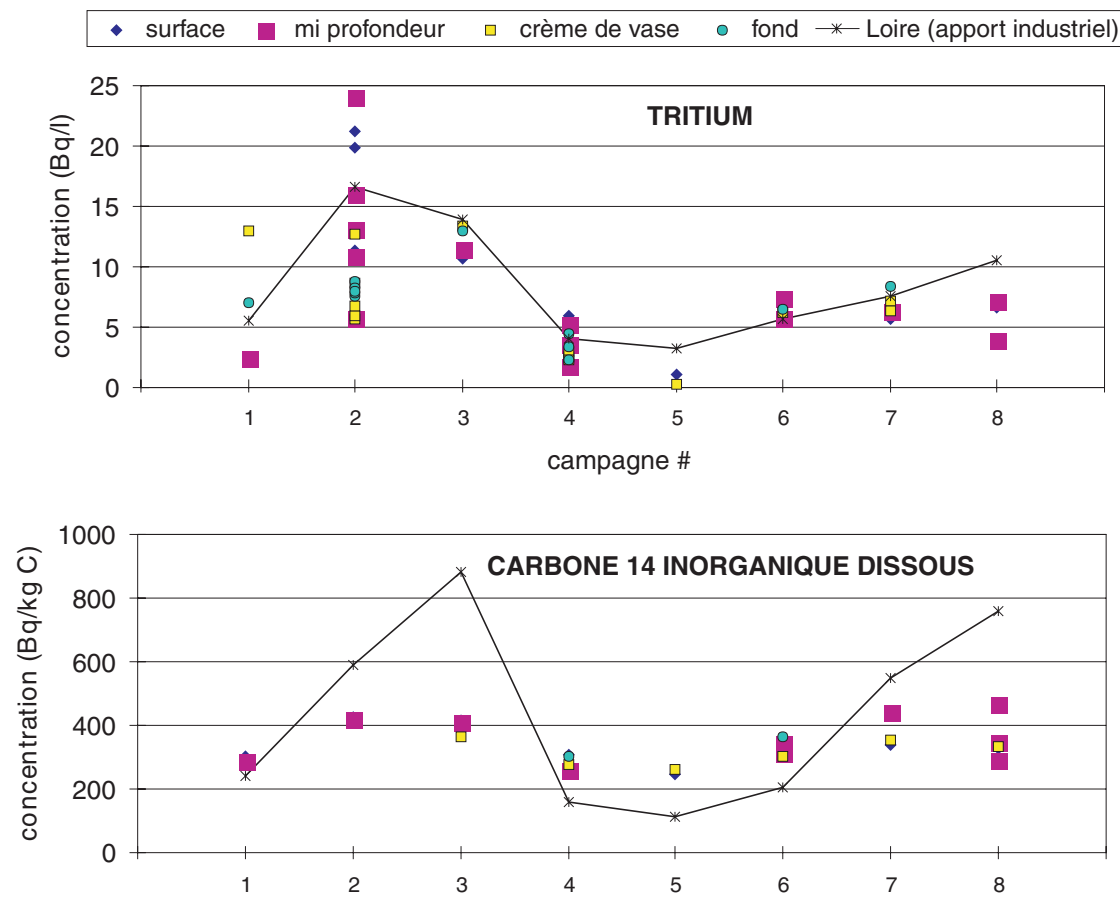

Fig. 19. - Concentrations en ${ }^{14} \mathrm{C}$ et ${ }^{3} \mathrm{H}$ mesurées dans le bouchon vaseux et apport par la Loire calculé avec CRESCENDO à partir des rejets des centrales. (Se référer au Tableau 3 pour les détails sur les campagnes de prélèvement).

Fig. 19. - Observed ${ }^{14} \mathrm{C}$ and ${ }^{3} \mathrm{H}$ concentrations in the turbidity maximum and calculated Loire input from nuclear power plant releases. (See Table 3 for details on sampling surveys).

particulaire restent relativement stables sur toute la plage de salinité. Les Kd observés dans l'estuaire varient de $200 \mathrm{l} / \mathrm{g}$ aux salinités proches de l'eau douce à $4 \mathrm{l} / \mathrm{g}$ en zone marine.

L'évolution des concentrations en ${ }^{90} \mathrm{Sr}$ en fonction de la salinité présente une tendance générale inverse de celle du ${ }^{137} \mathrm{Cs}$. Les teneurs les plus basses sont observées dans la référence océanique avec $1,4 \mathrm{~Bq} / \mathrm{m}^{3}$, alors qu'elles sont de l'ordre de $4,5 \mathrm{~Bq} / \mathrm{m}^{3}$ pour des salinités très faibles. Les calculs des apports liés aux rejets des centrales fournissent une valeur moyenne de ${ }^{90} \mathrm{Sr}$ dissous de $0,015 \mathrm{~Bq} / \mathrm{m}^{3}$, inférieure de plus de deux ordres de grandeur aux concentrations mesurées. Comme pour le ${ }^{90} \mathrm{Sr}$ particulaire, l'écart important entre les apports par les centrales et les valeurs observées montrent que les retombées des tirs nucléaires restent la principale origine de ce radionucléide. Les concentrations mesurées sont d'ailleurs voisines de celles observées dans des zones non soumises aux rejets nucléaires indus- 


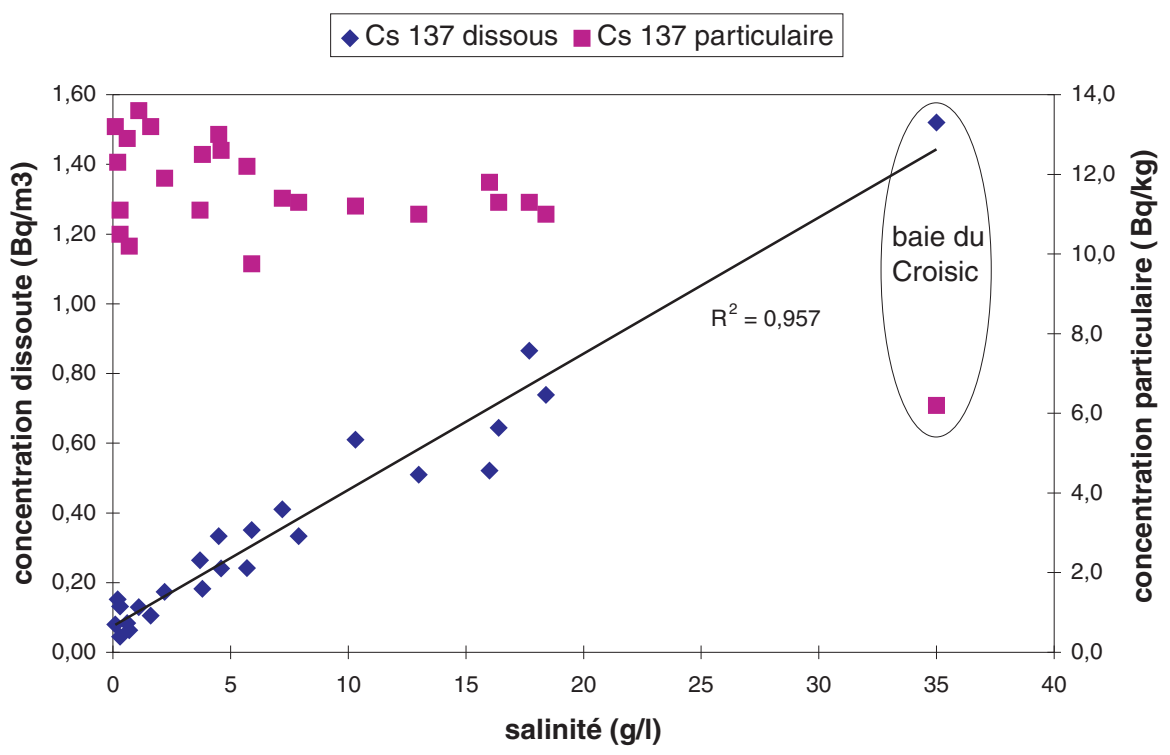

Fig. 20. - Evolution des concentrations en ${ }^{137} \mathrm{Cs}$ en fonction de la salinité. L'échantillon prélevé dans la baie du Croisic, située hors influence de l'estuaire, tient lieu de référence marine.

Fig. 20. - Evolution of Cs concentrations with salinity. The sea water sample collected in the Le Croisic Bay, located out of influence of the estuary, served as a marine reference.

triels (Tableau 5). Contrairement au ${ }^{137} \mathrm{Cs}$, il apparaît que les apports marins de ${ }^{90} \mathrm{Sr}$ sont inférieurs aux apports continentaux. Les valeurs de Kd observés dans l'estuaire sont peu nombreuses (six), en raison des difficultés à disposer sur un même échantillon de suffisamment d'eau et de solides pour mener à bien une analyse quantitative ; les $\mathrm{Kd}$ sont faibles et varient de $0,06 \mathrm{l} / \mathrm{g}$, pour des salinités moyennes, à 0,2 l/g pour de faibles salinités.

\subsection{Modélisation du transfert des radionucléides dans l'estuaire}

Afin de mieux comprendre les processus de sorption et de désorption des radionucléides dans le milieu estuarien, où les concentrations de salinité et de MES changent très rapidement, un modèle de transport à plusieurs variables a été développé à partir d'un modèle hydrodynamique existant de l'estuaire de la Loire. II représente la propagation de la marée, l'intrusion saline, le transport des radionucléides et des sédiments en suspension, la sédimentation et l'érosion, ainsi que la cinétique des échanges de radionucléides entre les formes dissoutes et particulaires.

On a procédé en laboratoire à des expériences sur la cinétique de sorption et de désorption des éléments $\mathrm{Co}, \mathrm{Mn}, \mathrm{Cs}, \mathrm{Ag}$ et $\mathrm{Sr}$ avec des échantillons d'eau provenant du fleuve et de son estuaire. Les valeurs de $\mathrm{Kd}$ à 
Tableau 5. - Teneurs en ${ }^{90} \mathrm{Sr}$ dans les eaux de surface continentales et océaniques.

Table 5. $-{ }^{90} \mathrm{Sr}$ activities in continental and oceanic surface waters.

\begin{tabular}{|c|c|c|c|}
\hline $\begin{array}{c}\text { Localisation } \\
\text { du prélèvement }\end{array}$ & $\begin{array}{c}\text { Nature de l'échantillon } \\
\text { et année de prélèvement }\end{array}$ & ${ }^{90}$ Sr en $\mathbf{~ B q} / \mathbf{m}^{3}$ & Référence \\
\hline Civaux & eau de Vienne 1996 & $9,5 \pm 0,9$ & [point zéro civaux] \\
\hline Civaux & eau de Vienne 1997 & $5,8 \pm 0,8$ & [point zéro civaux] \\
\hline Belleville & eau de Loire 1984 & 5 à 8,5 & [point zéro belleville] \\
\hline Golfech & eau de Garonne 1986 & 5 à 6 & [point zéro golfech] \\
\hline Golfech & eau de Garonne 1987 & 5 à 6 & [point zéro golfech] \\
\hline Finlande & eaux de rivière -1994-1996 & 4 à 8 & [10] \\
\hline Océan Atlantique Nord & eau de mer 1992-1994 & 1,2 à 1,6 & [11] \\
\hline
\end{tabular}

Tableau 6. - Kd à l'équilibre calculés à partir des cinétiques expérimentales.

Table 6. - Equilibrium Kd derived from experimental kinetics.

\begin{tabular}{|l|c|c|c|}
\hline & \multicolumn{3}{|c|}{ Kd en $\mathrm{l} / \mathrm{g}$} \\
\hline radionucléide & salinité $0 \%$ salinité $5 \%$ salinité $10 \%$ 。 & 28 \\
\hline argent & 808 & 150 & 315 \\
\hline cobalt & 1279 & 630 & 3 \\
\hline césium & 77 & 13 & \\
\hline
\end{tabular}

l'équilibre, dérivées des cinétiques expérimentales, sont présentées dans le Tableau 6 pour différentes salinités.

A partir des résultats obtenus, on a développé et étalonné un modèle cinétique constitué de deux processus d'échange successifs, le premier, rapide, entre la phase liquide et les sites de surface à faible affinité et le second, plus lent, entre les sites à faible affinité et ceux à forte affinité, avec des vitesses d'échange liées à la salinité pour les deux processus [4]. II a été intégré au modèle hydrodynamique et sédimentologique TELEMAC.

On a ensuite procédé à des simulations de courte durée avec le modèle TELEMAC afin d'observer les processus de sorption et de désorption sur quelques marées [5]. Les radionucléides étaient au départ considérés comme associés aux seules particules. Les résultats ont montré la même tendance générale pour le césium, le cobalt et l'argent : la désorption se produit immédiatement et jusqu'à ce que soit atteint le Kd expérimental à une salinité donnée (une heure après le début de la simulation).

Par la suite, les formes dissoutes et particulaires respectent les fluctuations du Kd en fonction de la salinité. Il est donc apparu qu'il n'était pas nécessaire de prendre en compte la cinétique des échanges, et que le $\mathrm{Kd}$ d'équilibre (Tableau 6) représentait correctement la séparation entre les formes dissoutes et particulaires. 
Pour des raisons numériques, il n'était pas possible d'appliquer ce modèle hydro-sédimentaire spatial détaillé à des simulations de longue durée. On a donc développé un modèle simplifié de type boîte, baptisé MOBHYDYS (MOdèle Boîte pour l'HYdro-DYnamique Sédimentaire) (Fig. 21). Ce modèle considère :

- les radionucléides pénétrant à l'intérieur de l'estuaire sont instantanément dilués dans le compartiment eau, et les échanges avec les matières en suspension se font en fonction du Kd ;

- le débit net maritime sortant à l'aval est égal au débit fluvial entrant à l'amont ;

- la quantité de particules et de radionucléides sous forme particulaire remobilisables et exportés vers l'océan est fonction de l'historique du débit fluvial (pris sur une période de 30 jours) selon la formule déterminée par Ciffroy [6] à partir de la datation au ${ }^{7} \mathrm{Be}$.

Les données d'entrée relatives aux apports de la Loire sont les résultats du modèle CRESCENDO (débit, concentrations en MES, concentrations en radionucléides dissous et particulaires), qui ne prend en compte que les rejets industriels de radionucléides.

La construction et le calage de ce modèle sont décrits de manière détaillée dans Siclet et al. [8].

Les simulations réalisées avec un pas de temps journalier sur la période 1994-1999 (Fig. 22) ont permis d'évaluer la masse sédimentaire moyenne à 700000 tonnes, pour un volume

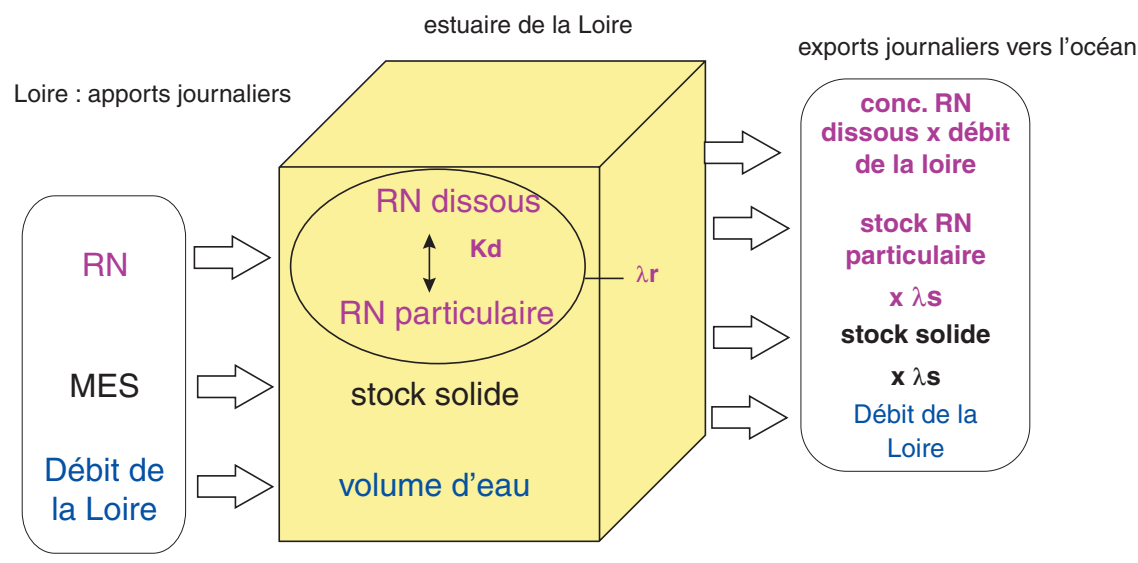

avec

$\mathrm{RN}$ : radionucléide

$\lambda r$ : constante de décroissance radioactive

$\lambda s$ : taux de perte par expulsion des particules hors de l'estuaire, basé sur l'approche ${ }^{7} B e$

Fig. 21. - Schéma descriptif du modèle MOBHYDYS.

Fig. 21. - Structure of the MOBHYDYS model 
d'eau constant de l'estuaire schématique, calé à 480 millions de $\mathrm{m}^{3}$. Le transport vers l'océan variait entre une valeur moyenne de 2800 tonnes par jour pour les débits inférieurs au module, jusqu'à 10000 tonnes par jour pour des débits de $2000 \mathrm{~m}^{3} / \mathrm{s}$. La totalité des sédiments était expulsée vers l'océan à des débits supérieurs à $5000 \mathrm{~m}^{3} / \mathrm{s}$.

Le comportement des radionucléides à forte affinité pour les MES suit celui de la masse sédimentaire (Fig. 23), tout en présentant des amplitudes de variations plus importantes liées aux variations dans le temps des flux de radionucléides apportés par les centrales. Ainsi pour le cobalt 60 présenté ici, les rejets les plus élevés de la période étudiée correspondent à l'année 1994 avec
6,7 GBq pour l'ensemble des centrales de Loire, ils diminuent ensuite progressivement chaque année jusqu'en 1998 avec une valeur de 2,7 GBq avant d'augmenter légèrement en 1999.

Le temps de résidence moyen varie de 10 jours pour un radionucléide à l'état dissous, comme le tritium, à 4,5 mois pour le cobalt 60 , d'une affinité forte pour les particules sédimentaires. II reste cependant inférieur à celui des particules égal à 7,4 mois, car la décroissance radioactive d'une part et la désorption même limitée d'autre part entraîne une disparition accrue des radionucléides, par rapport aux particules auxquelles ils sont associés.

Pour les radionucléides ayant plusieurs origines, les concentrations dans l'estuaire résultant des rejets

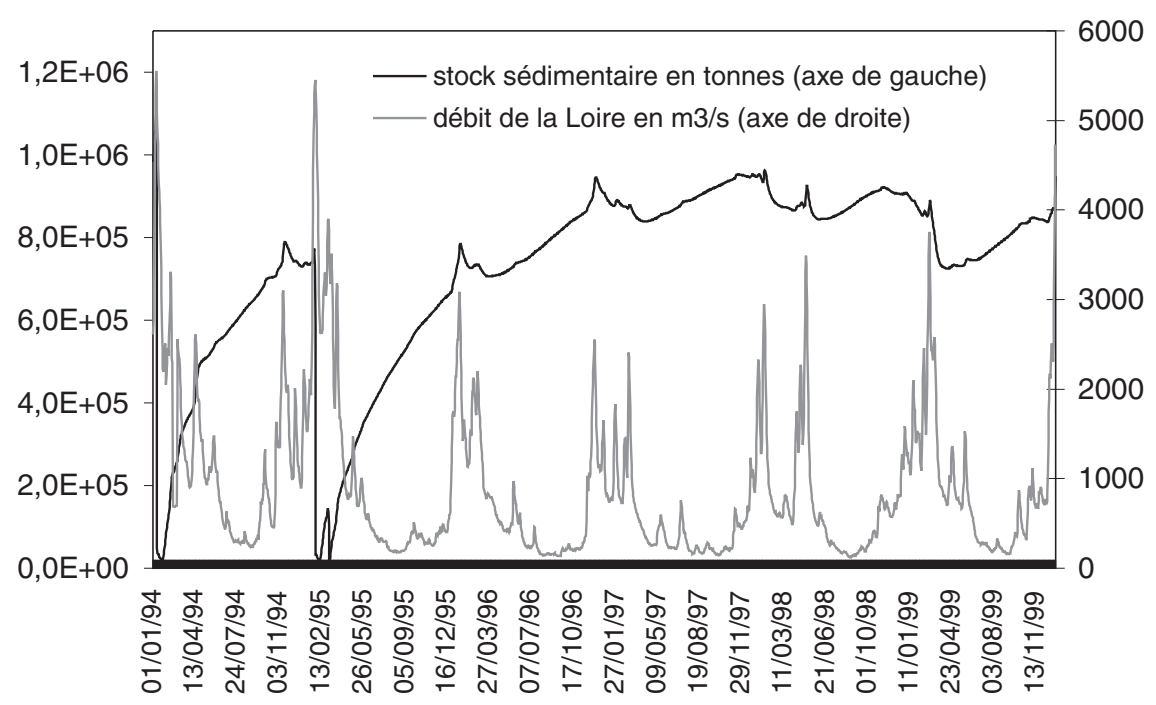

Fig. 22. - Evolution du stock de sédiment estuarien sur une période de six ans.

Fig. 22. - Evolution of estuarine sediment stock over a six-year period. 


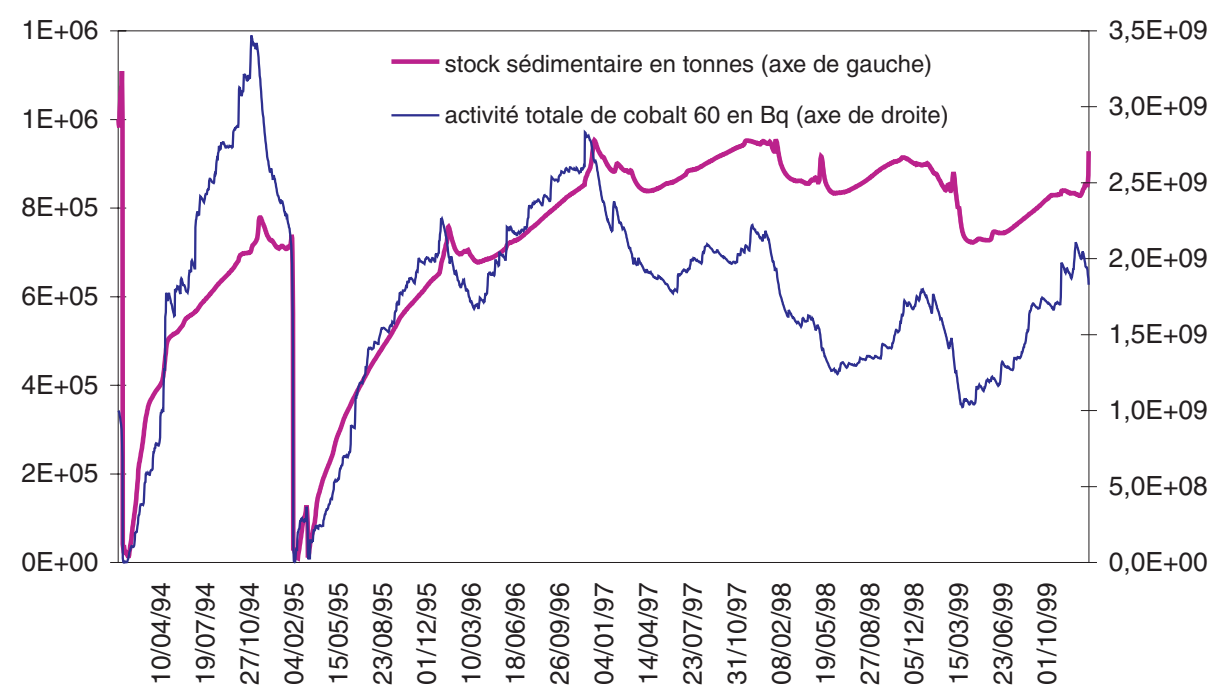

Fig. 23. - Evolution de l'activité totale en ${ }^{60} \mathrm{Co}$ présent dans l'estuaire. Comparaison avec le stock sédimentaire.

Fig. 23. - Evolution of ${ }^{60} \mathrm{Co}$ estuarine stock. Comparison with sediment stock.

radioactifs ont été calculées avec le modèle boîte, et comparées aux concentrations observées afin de calculer la contribution de la source industrielle. Cette approche a mis en évidence pour le césium 137 la nécessité de tenir compte des apports marins pour expliquer les niveaux observés dans les MES (Fig. 24). Le modèle MOBHYDYS a été modifié pour y inclure des échanges d'eau avec l'océan, basés sur des volumes oscillants calculés en fonction du coefficient de marée. Le césium océanique qui entre dans l'estuaire principalement sous forme dissoute, disparaît en partie de la phase liquide par sorption sur les particules du bouchon vaseux, et entraîne ainsi une augmentation substantielle de la ra- dioactivité stockée dans l'estuaire. D'après les simulations, $90 \%$ du stock en ${ }^{137} \mathrm{Cs}$ sous forme particulaire provient de l'océan. Le même phénomène a été mis en évidence pour le plutonium dans l'estuaire de la Savannah River [9]. Le bilan annuel des entrées et sorties de l'estuaire du ${ }^{137} \mathrm{Cs}$, sous forme dissoute et particulaire, correspond néanmoins à un export du bassin de la Loire vers l'océan. Sur la période étudiée, l'export net annuel varie de $2,7 \mathrm{GBq}$ de ${ }^{137} \mathrm{Cs}$ en 1997, année du plus faible débit moyen annuel en Loire, à 13,6 GBq en 1994, année du débit moyen le plus élevé. Les mêmes années, les rejets annuels des centrales s'élevaient à $0,8 \mathrm{GBq}$ et $3,2 \mathrm{GBq}$, respectivement. 


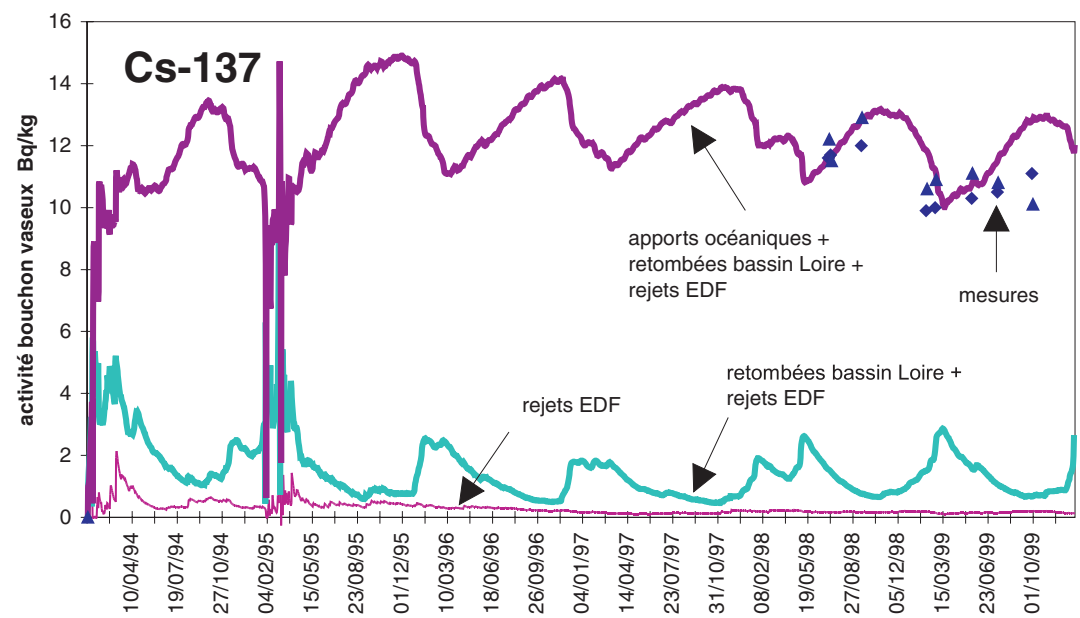

Fig. 24. - Comparaison entre les concentrations en ${ }^{137} \mathrm{C}$ s mesurées dans l'estuaire et celles calculées avec MOBHYDYS à partir des rejets des centrales, des retombées sur le bassin de la Loire, et des apports océaniques.

Fig. 24. - Comparison between observed ${ }^{137} \mathrm{Cs}$ concentrations and MOBHYDYS calculations taking into account power plant discharges, fallout on the Loire river watershed and oceanic input.

\subsection{Inventaire global de la radioactivité dans l'estuaire}

L'inventaire global présenté sur la Figure 25 est calculé en se basant sur:

- les moyennes des mesures de concentrations dans la phase particulaire d'une part (§ 3.2.1), et dans la phase dissoute d'autre part (§ 3.3),

- un volume d'eau de l'estuaire fixé à 480 millions de $\mathrm{m}^{3}$, d'après le calage de MOBHYDYS,

- une masse sédimentaire moyenne sur 1998-1999 de 835000 tonnes, issue des calculs avec MOBHYDYS.

Le tritium, issu des rejets des centrales EDF, est le radionucléide le plus abondant, présent essentiellement dans la phase soluble. II est sui- vi par les radionucléides naturels provenant de la croûte terrestre (familles de l'uranium et du thorium, et potassium 40) et le béryllium 7 d'origine cosmique, que l'on retrouve à $98 \%$ dans la masse particulaire.

Vient ensuite le carbone 14 avec un stock d'environ $10 \mathrm{GBq}$, dont $34 \%$ sous forme de carbone dissous. Ce radionucléide possède une triple origine : naturelle, militaire et industrielle. En considérant que le marquage de la phase particulaire a pour origines, d'une part la production naturelle de ${ }^{14} \mathrm{C}$, et d'autre part les retombées atmosphériques sur les bassins versants, alors que le marquage de la phase inorganique dissoute provient principalement de l'équilibre avec le $\mathrm{CO}_{2}$ atmosphérique et des rejets radioactifs liquides, et sachant que l'activité d'origine natu- 


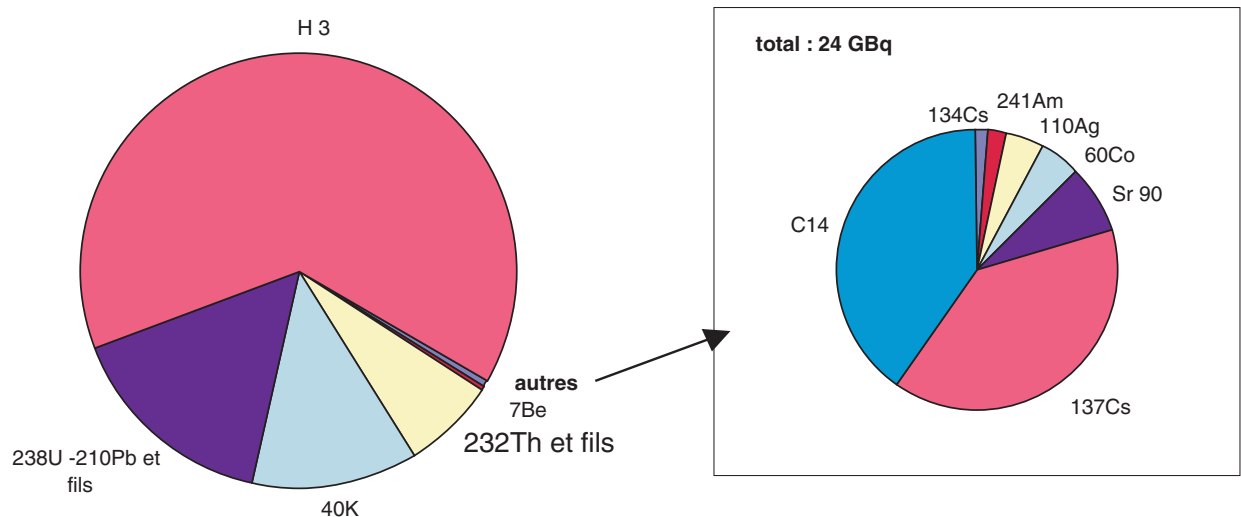

Fig. 25. - Inventaire de la radioactivité de l'estuaire de la Loire en 1998-1999.

Fig. 25. - Inventory of radioactivity in the Loire estuary in 1998-1999

relle en ${ }^{14} \mathrm{C}$ est de $226 \mathrm{~Bq} / \mathrm{kg}$ de carbone, la contribution des différentes sources est évaluée dans le Tableau 7 .

La modélisation du comportement du césium 137 avec MOBHYDYS a évalué la contribution des rejets EDF au stock de ${ }^{137} \mathrm{Cs}$ à $2 \%$. Le reste provient des retombées avec des apports par le bassin versant de la Loire et par l'océan. L'apport marin domine et représente $89 \%$ du stock présent dans l'estuaire. Les retombées de
Tchernobyl ont peu affecté le bassin de la Loire. Les mesures, réalisées dans le cadre de la surveillance radioécologique des centrales nucléaires, mettent en évidence à partir de 1987 un marquage, identifiable par la présence de césium 134, uniquement dans la partie la plus à l'Est du bassin versant. Sur la base de ces données, on peut estimer que, dans les zones les plus marquées, la contribution de l'accident de Tchernobyl aux dépôts de ${ }^{137} \mathrm{Cs}$ sur les sols est de $50 \%$, et

Tableau 7. - Origines du ${ }^{14} \mathrm{C}$ présent dans l'estuaire de la Loire.

Table 7. - Origins of Loire estuary ${ }^{14 \mathrm{C}}$.

\begin{tabular}{|l|c|c|c|}
\hline & \multicolumn{3}{|c|}{ Origines du ${ }^{14} \mathrm{C}$ de l'estuaire de la Loire } \\
\hline & naturelle & rejets & retombées \\
\hline${ }^{14} \mathrm{C}$ dissous & $65 \%$ & $31 \%$ & $5 \%$ \\
\hline${ }^{14} \mathrm{C}$ particulaire & $77 \%$ & $0 \%$ & $23 \%$ \\
\hline total & $72,7 \%$ & $10,5 \%$ & $16,7 \%$ \\
\hline
\end{tabular}


cela sur $20 \%$ du bassin versant, soit une contribution de $10 \%$ à l'apport fluvial de ${ }^{137} \mathrm{Cs}$, et de $1 \%$ au stock de ${ }^{137} \mathrm{Cs}$ dans l'estuaire de la Loire.

Le strontium 90 provient lui aussi essentiellement des retombées. Les activités mesurées montrent que $85 \%$ du stock de ${ }^{90} \mathrm{Sr}$ se trouve dans la phase dissoute. La concentration moyenne de $4 \mathrm{~Bq} / \mathrm{m}^{3}$, toutes formes confondues, est intermédiaire entre les niveaux rencontrés dans les eaux marines et les eaux continentales des zones soumises uniquement à l'influence des retombées (respectivement $1,4 \mathrm{~Bq} / \mathrm{m}^{3}$ et $\left.5 \mathrm{~Bq} / \mathrm{m}^{3}\right)$. Le marquage par les rejets des centrales, calculé à partir d'un terme-source majorant, basé sur la limite de détection de la mesure du strontium 90 , est inférieur à $1 \%$ du niveau en ${ }^{90} \mathrm{Sr}$ observé dans l'estuaire.

Les autres radionucléides rejetés par les centrales ne présentent qu'une contribution très faible à la radioactivité d'origine artificielle de l'estuaire, principalement avec le cobalt 60 et l'argent $110 \mathrm{~m}$, à hauteur de $1 \mathrm{GBq}$ (soit un dixième de la radioactivité due $a{ }^{137} \mathrm{Cs}$ ). Le cobalt 58 , l'antimoine 124 et le manganèse 54, présents dans les rejets, ne sont pas détectés au niveau de l'estuaire.

Les mesures montrent aussi la présence dans l'estuaire d'américium 241 (période radioactive : 462 ans), à l'état de traces, essentiellement associé aux particules. Ce radionucléide peut provenir des retombées directes des anciens tirs et de la désintégration du plutonium 241, lui aussi issu des retombées. On le trouve également dans les détecteurs de fumée et les paratonnerres. Sa concentration, en limite de détection dans l'estuaire, est du même ordre de grandeur que dans les eaux continentales ou dans l'océan.

\section{CONCLUSIONS}

Pour étudier le transfert des radionucléides et la dose correspondante due aux rejets périodiques des cinq centrales du bassin de la Loire, trois modèles principaux ont été élaborés, en s'appuyant sur les développements méthodologiques, les expérimentations et les campagnes de mesures réalisées dans le cadre du programme spécifique, mené par EDF, de 1998 à 2001 :

- CRESCENDO évalue avec un pas de temps horaire en tout point du bassin les concentrations en radionucléides dans les cours d'eau, en distinguant les formes dissoutes, particulaires et sédimentaires ;

- CALVADOS calcule avec un pas de temps mensuel les concentrations dans la chaîne alimentaire et les doses à l'homme liées à la présence de radionucléides dans les cours d'eau ;

- MOBHYDYS calcule avec un pas de temps journalier les concentrations moyennes en radionucléides dans l'estuaire et les flux exportés vers l'océan.

Les simulations réalisées à partir des rejets réels sur la période 1994 à 1999 montrent qu'à l'échelle du bassin, il n'y a pas de tendance générale 
à l'augmentation, de l'amont vers l'aval, des concentrations dans l'environnement et des doses à l'homme. On constate plutôt que, hormis les pics de concentrations à proximité des installations dans la zone de dilution partielle des effluents, les concentrations en radionucléides sous forme dissoute et particulaire restent relativement stables le long du cours d'eau, en raison des apports d'eau par les confluents qui compensent les apports de radionucléides par les rejets des centrales : les concentrations maximales (de l'ordre de $10 \mathrm{~Bq} / \mathrm{l}$ pour le tritium, de $10 \mathrm{~Bq} / \mathrm{m}^{3}$ pour le ${ }^{14} \mathrm{C}$ et de moins de $1 \mathrm{~Bq} / \mathrm{m}^{3}$ pour les autres radionucléides) sont observées dans le tronçon de $100 \mathrm{~km}$ entre Saint-Laurent et la confluence avec le Cher, en raison de la convergence des rejets de Belleville, Dampierre et Saint-Laurent, sans augmentation notable de la ressource en eau. Elles sont plus faibles d'un facteur deux, dans la section la plus en aval de la zone fluviale (de Saumur à Angers), avant la dispersion dans l'estuaire.

La distribution des radionucléides dans les sédiments de fond et sur les berges des cours d'eau est radicalement différente de celle dans la masse d'eau, avec une hétérogénéité spatiale importante liée à la topographie locale du lit de la rivière qui peut induire l'existence de zones calmes favorables à la sédimentation. Les accumulations de radionucléides dans les sédiments sont localisées notamment au niveau du barrage mobile de Blois, ainsi que sur les berges et bras morts ça et là.
Les concentrations en radionucléides calculées avec le modèle CRESCENDO concordent avec les valeurs observées, y compris en ce qui concerne les sédiments des rives. Toutefois, en raison des difficultés que pose la collecte de données précises sur l'épaisseur des dépôts de sédiments sur les rives de la Loire et de la Vienne, elles ne doivent être considérées comme réalistes que pour les zones limitées (quelques $\mathrm{dm}^{2}$ ) dans lesquelles un dépôt de particules fines peut réellement être observé. A une échelle plus large, les calculs surestiment certainement la moyenne des dépôts. II convient de souligner ce point, sachant que l'exposition qui en résulte intervient significativement dans l'évaluation de la dose.

Dans la masse turbide de l'estuaire de la Loire, on constate une diminution des concentrations par rapport aux MES présentes en Loire, diminution liée à la décroissance radioactive et à la désorption des radionucléides Iorsque la salinité augmente. Cependant, cette diminution d'activité des particules intervient en même temps qu'une forte augmentation de la turbidité, la conjugaison des deux phénomènes se traduisant globalement par une augmentation de l'activité totale (dissoute + particulaire), exprimée en $\mathrm{Bq} / \mathrm{m}^{3}$ d'eau, entre l'eau douce et l'estuaire. L'évolution du stock turbide dans l'estuaire va alors gouverner l'évolution du stock de radionucléides. La dynamique sédimentaire est elle-même contrôlée par le débit de la Loire avec une expulsion totale du 
bouchon vaseux uniquement pour des débits fluviaux très élevés (supérieurs à $5000 \mathrm{~m}^{3} / \mathrm{s}$ ) dont l'occurrence est faible ( 1 fois tous les 5 ans). II existe cependant une expulsion, faible mais continue, de particules vers l'océan qui limite l'accumulation dans l'estuaire. Les simulations réalisées sur la période 1994 à 1999 mettent en évidence un temps de résidence moyen des particules de 222 jours. Celui des radionucléides lui est inférieur (133 jours pour ${ }^{60} \mathrm{Co}$ ), en raison des pertes par décroissance radioactive et désorption. II y a donc bien une accumulation, dans le bouchon vaseux, des radionucléides à forte affinité pour les particules, mais elle est limitée (moins de $30 \%$ des rejets annuels des centrales). Les calculs d'inventaire de la radioactivité sous forme particulaire montrent d'autre part que la contribution des radionucléides d'origine naturelle (potassium 40, béryllium 7, famille de l'uranium 238 et du thorium 232) dépassent d'un facteur 1000 celle provenant des rejets industriels. Quant aux retombées des tirs nucléaires militaires des années soixante, leur contribution est, encore de nos jours, supérieure d'un facteur 5 à celle des centrales.

Les doses à l'homme dues à l'ensemble des rejets radioactifs liquides des 5 centrales du bassin, calculées avec CALVADOS à partir des résultats de CRESCENDO, restent très basses: elles varient de 0,06 à 1,7 microsievert par an en fonction des stations (limite recommandée pour le public de 1000 microsievert par an). Les voies d'exposition et les radionucléides responsables de l'essentiel de la dose au public sont l'ingestion de tritium dans l'eau de boisson, de carbone 14 dans les poissons, et l'exposition externe au cobalt 60 et à l'argent $110 \mathrm{~m}$ des sédiments des berges.

L'ensemble de ces résultats, qu'on les compare à la radioactivité d'origine naturelle ou aux limites réglementaires, confirment l'effet extrêmement faible des rejets radioactifs des centrales sur l'environnement et l'homme, à l'échelle du bassin de la Loire.

\section{REFERENCES BIBLIOGRAPHIQUES}

[1] Reyss (J.L.), Le Petit (G.) \& Siclet (F.), 2001 - ${ }^{137} \mathrm{Cs}$ and ${ }^{90} \mathrm{Sr}$ in the estuarine turbidity maximum of the Loire river. Ecorad 2001, Aix-en-Provence, Sept 3-7, 2001.

[2] Fontugne (M.), Sadouni (N.) \& Saliot (A.), $2002-{ }^{14} \mathrm{C}$ activity in dissolved mineral carbon and identified organic matter in the Loire estuary (France). Ecorad 2001, Aix-en-Provence, Sept 3-7, 2001. Radioprotection - Colloques 37, C1 775 - 780.

[3] Reyss (J.L.), Siclet (F.) \& Cazala (C.), 2002 - Sediment records in the river Loire Estuary. Ecorad 2001, Aix-enProvence, Sept 3-7, 2001. Radioprotection - Colloques 37, C1 769- 773.

[4] Ciffroy (P.), Garnier (J.M.) \& Benhyahya (L.), 2001 - Adsorption/desorption kinetics of $\mathrm{Co}, \mathrm{Mn}, \mathrm{Cs}, \mathrm{Fe}, \mathrm{Ag}, \mathrm{Zn}$ and $\mathrm{Cd}$ in the Loire and the macro-tidal Loire estuary : experimental and modelling approaches. Ecorad 2001, Aixen-Provence, Sept 3-7, 2001. 
[5] Guesmia (M.), Reyss (J.L.) \& Cheviet (C.) 2002 - Modelling of ${ }^{60} \mathrm{Co}$ concentrations in the dissolved and particulate phases in the Loire estuary (France). Ecorad 2001, Aix-en-Provence, Sept 3-7, 2001, Radioprotection-Colloques 37, C1 781- 787.

[6] Ciffroy (P.), Reyss (J.L.) \& Siclet (F.), 2003 - Determination of the residence time of suspended particles in the turbidity maximum of the Loire estuary by ${ }^{7} \mathrm{Be}$ analysis. Estuarine, Coastal and Shelf Science 56,1-16.

[7] Jouanneau (J.M.), 1987 - The contribution of ${ }^{14} \mathrm{C}$ dating to a better understanding of the POM behaviour in estuaries. Marine Chemistry 21, 189197.

[8] Siclet (F.), Guesmia (M.), Ciffroy (P.), Reyss (J.L.), Fontugne (M.), Lepetit (G.), Jean-Baptiste (P.) \& Drouadaine (L.), 2001 - Radionuclides in the Loire river estuary (France) : sources, transport and fate. Ecorad 2001, Aix-enProvence, Sept 3-7, 2001. Radioprotection-Colloques 37, C1 761- 767.
[9] Olsen (C.R.), Thein (M.), Larsen (I.L.), Lowry (P.D.), Mulholland (P.J.), Cutshall (N.H.), Byrd (J.T.) \& Windom (H.L.), 1989 - Plutonium, lead-210, and carbon isotopes in the Savannah Estuary: riverborne versus marine sources. Environ. Sci. Technol.23, 1475-1481.

[10] Saxen (R.) \& Ilus (E.), 2001 - Discharge of ${ }^{137} \mathrm{Cs}$ and ${ }^{90} \mathrm{Sr}$ by Finnish rivers to the Baltic Sea in 1986-1996. J. Environ. Radioactivity 54, 275-291.

[11] Bourlat (Y.), Millies-Lacroix (J.C.), Le Petit (G.) \& Bourguignon (J.), 1996 ${ }^{90} \mathrm{Sr},{ }^{137} \mathrm{Cs}$ and ${ }^{239+240} \mathrm{Pu}$ in world ocean water samples collected from 1992 to 1994. In Radionuclides in the Oceans - Inputs and inventories. Les éditions de physique, Les Ulis, France, collection IPSN. pp 75-93.

[12] Ciffroy (P.), Garnier (J.M.) \& Pham (M.K.), 2001 - Kinetics of Co, Mn, Fe, $\mathrm{Ag}$ and $\mathrm{Cd}$ adsorption in freshwater: experimental and modelling approach. J. Environ. Radioactivity 55, 71-91. 
